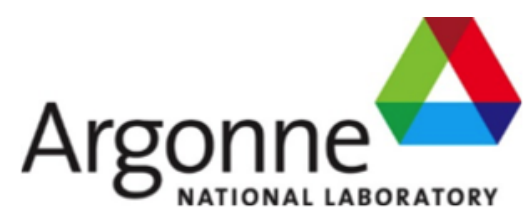

ANL-ART-195

\title{
Initial High Temperature Inelastic Constitutive Model for Alloy 617
}

Applied Materials Division 
About Argonne National Laboratory

Argonne is a U.S. Department of Energy laboratory managed by UChicago Argonne, LLC under contract DE-AC02-06CH11357. The Laboratory's main facility is outside Chicago, at 9700 South Cass Avenue, Argonne, Illinois 60439. For information about Argonne and its pioneering science and technology programs, see www.anl.gov.

\section{DOCUMENT AVAILABILITY}

Online Access: U.S. Department of Energy (DOE) reports produced after 1991 and a growing number of pre-1991 documents are available free at OSTI.GOV (http://www.osti.gov/), a service of the U.S. Dept. of Energy's Office of Scientific and Technical Information

Reports not in digital format may be purchased by the public from the National Technical Information Service (NTIS):

U.S. Department of Commerce

National Technical Information Service

5301 Shawnee Rd

Alexandria, VA 22312

www.ntis.gov

Phone: (800) 553-NTIS (6847) or (703) 605-6000

Fax: (703) 605-6900

Email: orders@ntis.gov

Reports not in digital format are available to DOE and DOE contractors from the Office of Scientific and Technical Information (OSTI)

U.S. Department of Energy

Office of Scientific and Technical Information

P.O. Box 62

Oak Ridge, TN 37831-0062

www.osti.gov

Phone: (865) 576-8401

Fax: (865) 576-5728

Email: reports@osti.gov

\section{Disclaimer}

This report was prepared as an account of work sponsored by an agency of the United States Government. Neither the United States Government nor any agency thereof, nor UChicago Argonne, LLC, nor any of their employees or officers, makes any warranty, express or implied, or assumes any legal liability or responsibility for the accuracy, completeness, or usefulness of any information, apparatus, product, or process disclosed, or represents that its use would not infringe privately owned rights. Reference herein to any specific commercial product, process, or service by trade name, trademark, manufacturer, or otherwise, does not necessarily constitute or imply its endorsement, recommendation, or favoring by the United States Government or any agency thereof. The views and opinions of document authors expressed herein do not necessarily state or reflect those of the United States Government or any agency thereof, Argonne National Laboratory, or UChicago Argonne, LLC. 
ANL-ART-195

\section{Initial High Temperature Inelastic Constitutive Model for Alloy 617}

Applied Materials Division

Argonne National Laboratory

August 2020

Prepared by

M. C. Messner, Argonne National Laboratory

T.-L. Sham, Argonne National Laboratory 



\section{Abstract}

This report describes progress on a Alloy 617 inelastic constitutive model slated for inclusion in the ASME Boiler \& Pressure Vessel Code in Code Case N-898 "Use of Alloy 617 (UNS N06617) for Class A Elevated Temperature Construction, Section III, Division 5" as part of an appendix on inelastic modeling. This appendix will provide a reference material model for A617 for use in conjunction with the ASME design by inelastic analysis criteria. This report focuses on the implementation and evaluation of an existing Alloy 617 model developed to represent the data collected at Idaho National Laboratory (INL) and Oak Ridge National Laboratory (ORNL) as part of the Alloy 617 Code Case experimental campaign. While the model accurately captures the cyclic stress/strain behavior of Alloy 617, it fails to capture the long-term creep and lower temperature tensile data. In addition, the model form is complicated and has over 50 configurable parameters. As such, we recommend the development of a simplified model, incorporating key features of the ORNL model but reducing the model complexity. 

Initial High Temperature Inelastic Constitutive Model for Alloy 617

August 2020

\section{Table of Contents}

Abstract i i

Table of Contents $\quad$ iii

List of Figures $\quad$ V

List of Tables vii

1 Introduction 1

1.1 Objectives . . . . . . . . . . . . . . . . . . . . 1

1.2 Organization of the report . . . . . . . . . . . . . . . . 3

2 The Walker A617 model 5

2.1 Original model . . . . . . . . . . . . . . . . . . . . 5

2.2 Implementation in NEML . . . . . . . . . . . . . . . . . 6

2.2.1 Basic viscoplastic response . . . . . . . . . . . . . . . . 6

2.2.2 Modification for a rate-insensitive response . . . . . . . . . . . . . . . . . 9

2.3 Parameter set identification and verification . . . . . . . . . . . 11

3 Model assessment 21

3.1 Comparison to data . . . . . . . . . . . . . . . . . . 21

3.1.1 Rate sensitivity via Kocks-Mecking diagrams . . . . . . . . . . . . . . 21

3.1 .2 Flow curves . . . . . . . . . . . . . . . . . . . 21

3.1.3 Creep data . . . . . . . . . . . . . . . . . . . . . . . . . . . . . . . . . . . . . . . 22

3.1.4 Strain-controlled cyclic tests . . . . . . . . . . . . . . 28

3.2 Assessment . . . . . . . . . . . . . . . . . . . . . . . . 30

4 Conclusions 33

Acknowledgments

$\begin{array}{ll}\text { Bibliography } & 37\end{array}$ 



\section{List of Figures}

2.1 Flow curves from four different model implementations at three different strain rates demonstrating the effectiveness of Walker's method for transitioning from a viscoplastic, rate-dependent response to a rate-insensitive response approximating classical rate independent plasticity. The three strain rates applied were $10^{-6}, 10^{-4}$, and $10^{-2} 1 / \mathrm{s}$. The dotted lines giving the $\lambda=0.99$ results all overlap, i.e. this model gives the same response regardless of the applied strain rate. . . . . . . . . . . . . . . .

2.2 Comparison between the model results and a fully-reversed strain-controlled cyclic experiment at $950^{\circ} \mathrm{C}$ for a total strain range of $1 \%$ and a 10 minute hold time. The solid lines are the model results, the dashed lines are experiments.

3.1 Kocks-Mecking diagram for Alloy 617 comparing the experimental data to the Walker model for all four parameter sets. . . . . . . . . . . . . . . . .

3.2 Comparison between the model predictions and a collection of uniaxial tension curves collected from INL experimental data. Solid lines are model predictions, dashed lines are corresponding experimental data. The line colors correspond to the test temperature. . . . . . . . . . . . . . . . . . . 23

3.3 Comparison between parameter set "A" and the creep test data. . . . . . . . 24

3.4 Comparison between parameter set "B" and the creep test data. . . . . . . . 25

3.5 Comparison between parameter set "C" and the creep test data. . . . . . . 26

3.6 Comparison between parameter set "D" and the creep test data. . . . . . . . 27

3.7 Detailed comparison between model results for parameter set "A" (black) and the reference creep-fatigue test (red). Subfigure (a) shows the comparison between the stress/strain hysteresis loops and subfigure (b) shows the comparison as a plot of stress versus time. (a) shows the data for all 100 cycles, (b) shows a truncated time series to highlight the stress relaxation profile over the first few cycles. . . . . . . . . . . . . . . . . . . . .

3.8 Detailed comparison between model results for parameter set "B" (black) and the reference creep-fatigue test (red). Subfigure (a) shows the comparison between the stress/strain hysteresis loops and subfigure (b) shows the comparison as a plot of stress versus time. (a) shows the data for all 100 cycles, (b) shows a truncated time series to highlight the stress relaxation profile over the first few cycles.

3.9 Detailed comparison between model results for parameter set "C" (black) and the reference creep-fatigue test (red). Subfigure (a) shows the comparison between the stress/strain hysteresis loops and subfigure (b) shows the comparison as a plot of stress versus time. (a) shows the data for all 100 cycles, (b) shows a truncated time series to highlight the stress relaxation profile over the first few cycles. 
3.10 Detailed comparison between model results for parameter set "C" (black) and the reference creep-fatigue test (red). Subfigure (a) shows the comparison between the stress/strain hysteresis loops and subfigure (b) shows the comparison as a plot of stress versus time. (a) shows the data for all 100 cycles, (b) shows a truncated time series to highlight the stress relaxation profile over the first few cycles. . . . . . . . . . . . . . . . . .

3.11 Comparison between the model and experimental maximum and minimum stress per cycle for three cyclic tests. The conditions shown in figure legends give the total strain range for fully-reversed, strain-controlled loading and the tensile hold time. All tests were at $950^{\circ} \mathrm{C} . \ldots \ldots$. . . . . . . . .

3.12 Comparison in average mean relative error for the four parameter sets, evaluated over all 32 strain controlled cyclic experiments. . . . . . . . . . . . 


\section{List of Tables}

2.1 Fixed, temperature-independent parameters for all four model. . . . . . . . . 12

2.2 Temperature-dependent rate switching parameter values and other basic data. All four parameter sets have the same values. The italicized coefficients are estimated. . . . . . . . . . . . . . . . . . . .

2.3 Temperature-dependent parameters for parameter set "A". Parameters with descriptions in italics are constant in temperature. . . . . . . . . . . . . .

2.4 Temperature-dependent parameters for parameter set "B". Parameters with descriptions in italics are constant in temperature. . . . . . . . . . . . . 15

2.5 Temperature-dependent parameters for parameter set "C". Parameters with descriptions in italics are constant in temperature. . . . . . . . . . . 16

2.6 Temperature-dependent parameters for parameter set "D". Parameters with descriptions in italics are constant in temperature. . . . . . . . . . . . 17 



\section{Introduction}

\subsection{Objectives}

This report describes the preliminary development of a high temperature inelastic constitutive model for Alloy 617, suitable for use with the Section III, Division 5, Subsection HB, Subpart B ASME Boiler \& Pressure Vessel Code design by inelastic analysis rules covering the design and construction of structural components in high temperature nuclear reactors. The model will be included in the Code as an appendix to Code Case N-898 "Use of Alloy 617 (UNS N06617) for Class A Elevated Temperature Construction, Section III, Division 5," which allows the use of Alloy 617 in elevated temperature Class A construction. The ASME Code and associated Code Cases offer several options for design and analysis methods: the classical design by elastic analysis, new design by elastic-perfectly plastic analysis methods, and design by inelastic analysis. The design by inelastic analysis method has a comparable history to the elastic analysis methods, and was used in the design of the Clinch River Breeder Reactor [1]. Of all the currently-available design methods, design by inelastic analysis produces the most efficient designs with the least amount of over-conservatism. However, it is the most expensive, in terms of analysis time, as it requires a full, detailed, cyclic inelastic analysis of the component. In the Clinch River project it was used to design the most challenging components, where the simpler and comparatively more conservative design by elastic analysis technique could not produce a viable design.

A critical component of the design by inelastic analysis method is a suitable inelastic constitutive model that describes the deformation of the material under the component operating conditions. The current, 2019 version of the ASME Code contains only very limited guidance on the construction of a suitable material model and does not provide reference model implementations for any of the Class A materials. These limitations are a substantial barrier to using design by inelastic analysis, as it puts the burden on either the plant owner or designer to develop or specify the inelastic models used in the plant design. Developing an adequate constitutive model and collecting the data required to produce an accurate model is a difficult task that historically was performed by the DOE research laboratories, and not the reactor designer, in the Clinch River Project.

This report is then one in a series of reports providing the technical basis for a new appendix ("Appendix Z") to the Section III, Division 5, Subsection HB, Subpart B rules including more detailed guidance on the construction of inelastic models and providing a reference implementation of a model for each Class A material. The particular model for Alloy 617 described in this report is a part of this general effort, though it will be implemented in Code Case N-898 rather than the base Code, as that Code Case contains the rules specific to Alloy 617. These reference implementations will not be mandatory - designers can still develop and use their own constitutive models - but they will be deemed acceptable for use with the design by inelastic analysis method without further validation by the designer. As such, this appendix will promote the use of the design by inelastic analysis method, giving designers better access to a more accurate and less over conservative design method.

Previous reports and journal publications $[2,3,4,5]$ cover the development of constitutive models for Grade 91 and 316H stainless steel. A previous report [6] also describes in detail a set of criteria for developing a model suitable for use with the ASME design method. In short, these criteria are: 
- The model must capture the cyclic stress/strain/time response of the material undergoing periodic loading intermixed with hold periods at constant stress or strain. This material response can be measured with strain-controlled (creep-fatigue) or stresscontrolled cyclic tests.

- A model should capture the average response of several heats of material.

- Models used in evaluating the ASME strain accumulation limits should account for primary, secondary, and tertiary creep. Models only used for creep-fatigue evaluation need only capture the response of the material before the onset of tertiary creep.

- The model must capture the interaction of plastic deformation on creep and vice-versa. Oftentimes this requires a unified viscoplastic formulation that treats both creep and plasticity with the same model.

- The model must account for the effect of stress multiaxiality and non-proportional loading.

- The model should be validated against experimental data at a wide variety of temperatures and under a wide variety of conditions. The model should capture the material response for all metal temperatures expected in the component operating life. The model should capture the rate sensitivity of the material through a reasonable range of strain rates, typical of those expected in service.

Developing a model that meets these criteria is not trivial. Even when adequate data can be collected from the literature and past work, developing the model form and calibrating the model is typically a dedicated research project.

This report describes the preliminary development of a model for Alloy 617. Alloy 617 is a high temperature Ni-based alloy slated for use in future very high temperature gas cooled reactors. This material was recently qualified for Section III, Division 5 use as a Class A material through Code Case N-898. This report focuses on evaluating current Alloy 617 constitutive models to establish their suitability for use with the ASME Code rules. In particular, this report focuses on a unified constitutive model developed at Oak Ridge National Laboratory (ORNL) in tandem with the experimental program at Idaho National Laboratory (INL) leading to the Alloy 617 Code Case. In addition, the report briefly describes data collection efforts to establish a model calibration/validation data set for fitting and evaluating models. This data collection effort focused mostly on standardizing the INL experimental data, however it did identify additional sources of data from the literature. One challenge with the experimental data is the relative lack of cyclic testing at lower temperatures. In particular, the INL cyclic tests focused only on a temperature of $950^{\circ} \mathrm{C}$.

The Alloy 617 model evaluated here was developed by Walker and Sham at ORNL. It has a unified viscoplastic form and contains terms aimed at capturing the required material behavior detailed above. This report focuses on implementing the model in a constitutive library (NEML) developed at Argonne National Laboratory (ANL) as part of the Appendix $\mathrm{Z}$ project and evaluating the model for Division 5 use by comparing it to the experimental database. The Walker model is quite detailed - it aims to capture the cyclic response of 
Alloy 617, including fine details of the material hysteresis - and consequently has a complicated mathematical form and many temperature-dependent parameters. The model captures the Alloy 617 creep-fatigue test data, particularly at the temperature $\left(950^{\circ} \mathrm{C}\right)$ where it was directly calibrated to experimental data. However, in other ways the model is less suitable. In particular it fails to capture the long-term creep behavior of Alloy 617. Furthermore, the model form is quite complicated and it has a large number of temperature-dependent material parameters. As such, we recommend the development of a simplified A617 model, retaining the critical features of the Walker model but simplifying the mathematical form of the model and reducing the number of model parameters. This new, simplified model should be recalibrated against experimental data with a focus on capturing the material's long-term creep response at least out to the start of tertiary creep.

\subsection{Organization of the report}

Chapter 2 describes the Walker model, the various parameter sets developed for the model during its development, the implementation of the model in NEML, and the verification of the NEML implementation. Chapter 3 describes the evaluation of the model for Section III, Division 5 use by comparison to the Alloy 617 experimental database. Finally, Chapter 4 summarizes the report and describes a plan for future work on the Alloy 617 inelastic model. 



\section{The Walker A617 model}

\subsection{Original model}

A conference paper [7] and direct communication with the original model developers [8] was used to reconstruct the model. The model form and parameter sets evolved during the development process. This report implements and evaluates the final version of the model, completed around 2014-2015.

Section 2.2 provides a mathematical description of the model, less a term designed to capture stress overshoots during cyclic tests (see below). This section focuses on the features of the deformation of Alloy 617 captured by the model to highlight why this model form and the calibrated parameters developed by Walker et al. over the course of the project could form a good basis for the Alloy 617 reference constitutive model.

The model was developed to capture most of the detailed, time-dependent deformation of Alloy 617, as quantified by an extensive series of tests completed at INL and ORNL:

- At high temperatures the model has a unified viscoplastic form to capture the interaction of creep and plasticity.

- The model has a mechanism to transition to a rate insensitive response at lower temperatures, where the experimental material flow stress does not depend on the strain rate and where creep is negligible.

- A Chaboche kinematic hardening model captures the basic stress- and strain-controlled cyclic behavior of the material.

- Flow softening functions are included in the basic viscoplastic rate and the internal variable evolution to capture the increase in creep rate at the onset of tertiary creep.

- The isotropic hardening formulation and drag stress evolution accounts for the cyclic softening observed in the INL experiments.

- Though, as described in the introduction, there is a lack of detailed experimental data for Alloy 617 at low temperatures (below the range tested as part of the INL experimental campaign), the model includes a thermal scaling function that allows it to reasonably represent the low temperature response of the material based on the available tension test data.

- The model has a dedicated mathematical submodel to capture the flow stress overshoot observed in the early cycles of cyclic tests at INL. However, as described below, the NEML implementation omits this submodel.

- Walker et al. developed several parameter sets for the model calibrated against the available experimental data. These parameter sets all produce slightly different responses, but all reasonably capture the average response of the material under the conditions of interest for high temperature reactor component design.

Walker explored several numerical integration methods. The final UMAT implementation had options for either an explicit Runge-Kutte method or an implicit exponential method 
using a quadratic fixed-point scheme to solve the resulting system of nonlinear equations without forming the exact Jacobian [9]. Walker also explored various integration schemes for stiff ODEs that require the material Jacobian. However, his implementation did not calculate this Jacobian analytically, and so these methods used a finite difference approximation. This significantly affected the numerical efficiency of the model.

The algorithmic tangent for the model was implemented using either finite differencing or an approximate algebraic relation. Both tangents were inexact, meaning the model did not converge quadratically when embedded in finite element simulations.

Walker used genetic algorithm optimization to calibrate the model to Alloy 617 data collected at INL and ORNL. The model calibration processes in the end led to four sets of material parameters labeled "A", "B", "C", and "D". This report implements and evaluates all four parameter sets.

\subsection{Implementation in NEML}

This reports describes the implementation of a variant of the Walker model in the NEML library (https://github.com/Argonne-National-Laboratory/neml). The implementation includes the complete Walker model except a term design to model the stress overshoot observed during the early part of creep-fatigue tests at INL [10]. This term was dropped in the NEML implementation because:

- This overshoot diminishes with repeated cycles and eventually disappears;

- Given that most engineering components experience many repetitive loading cycles, the overshoot phenomena is of limited importance for engineering design;

- The extra term adds six additional temperature-dependent material parameters.

The subsequent text splits the model description into two parts. The first part describes the base viscoplastic model and corresponding model parameters. The second part describes a modification Walker developed to take the basic viscoplastic response and reduce it to rateindependent plasticity at lower temperatures. As implemented in NEML, this modification can be applied to any viscoplastic model, not just the Walker A617 model.

\subsubsection{Basic viscoplastic response}

The model starts with the basic inelastic stress rate equation:

$$
\dot{\boldsymbol{\sigma}}=\boldsymbol{C}:\left(\dot{\varepsilon}-\dot{\varepsilon}_{v p}-\dot{\varepsilon}_{t h}\right)
$$

with $\dot{\boldsymbol{\sigma}}$ the stress rate, $\boldsymbol{C}$ an isotropic elasticity tensors described by Young's modulus $E$ and Poisson's ratio $\nu, \dot{\varepsilon}$ the total (applied) strain rate, $\dot{\varepsilon}_{v p}$ is the viscoplastic strain rate described below, and $\dot{\varepsilon}_{t h}$ is the thermal strain rate given by

$$
\dot{\varepsilon}_{t h}=\alpha \dot{T} \boldsymbol{I}
$$

with $\alpha$ the instantaneous coefficient of thermal expansion, $\dot{T}$ the temperature rate, and $\boldsymbol{I}$ the identity tensor. 
The model definition reuses several common functions. These functions appear several places in the formulation, occasionally with the same parameters and occasionally with different parameters depending on the location. Where needed this exposition differentiates the parameters using parenthetical superscripts, e.g. $x^{(a)}$.

\subsubsection{Temperature scaling function}

The temperature scaling function:

$$
\chi(T)=\frac{\exp \left(-\frac{Q}{R T}\right)}{\exp \left(-\frac{Q}{R T_{r e f}}\right)}
$$

with $Q$ an activation energy, $R$ the gas constant, and $T_{r e f}$ a reference temperature, with temperature in Kelvin, is reused several places in the model. The thermal scaling constants $Q$ and $T_{r e f}$ remain the same with each appearance and so no superscripts are required.

\subsubsection{Strain softening/tertiary creep function}

The tertiary creep function

$$
\Phi=1+\phi_{0} p^{\phi_{1}}
$$

with $p$ the equivalent plastic strain and $\phi_{0}$ and $\phi_{1}$ temperature-dependent constants likewise appears several times in the model. Different components of the model uses different parameters $\phi_{0}$ and $\phi_{1}$, differentiated by superscripts.

\subsubsection{The viscoplastic strain rate}

The viscoplastic strain rate is

$$
\dot{\varepsilon}_{v p}=\dot{p} \boldsymbol{g}
$$

where $\dot{p}$ is the scalar plastic strain rate and $\boldsymbol{g}$ the flow direction. The flow direction is

$$
\boldsymbol{g}=\sqrt{\frac{3}{2}} \frac{s-\boldsymbol{X}}{\|s-\boldsymbol{X}\|}
$$

with $s$ the deviatoric part of the stress, $s=\operatorname{dev}(\boldsymbol{\sigma})$ and $\boldsymbol{X}$ the backstress defined below. In this expression and in the equations below the tensor norm is defined as

$$
\|\boldsymbol{Y}\|=\sqrt{\boldsymbol{Y}: \boldsymbol{Y}}
$$

with : indicating double contraction.

The scalar strain rate is

$$
\dot{p}=\dot{\varepsilon}_{0} \Phi^{(p)}(p) \chi(T) F
$$

with $\dot{\varepsilon}_{0}, \Phi^{(p)}(p)$ a softening function defined by constants $\phi_{0}^{(p)}$ and $\phi_{1}^{(p)}, \chi(T)$ the temperature scaling function, and $F$ the flow function

$$
F=\left\langle\frac{\sqrt{3 / 2}\|s-X\|-Y}{D}\right\rangle^{n}
$$


with $D$ an internal variable defined later, $n$ a parameter, \langle\rangle the Macaulay brackets, and $Y$ the threshold stress given as

$$
Y=(k+R)\left(\frac{D-D_{0}}{D_{\xi}}\right)^{m}
$$

with $k, D_{0}, D_{\xi}$, and $m$ parameters and $R$ and internal variable defined below.

\subsubsection{Isotropic hardening}

The isotropic hardening variable evolves as

$$
\dot{R}=r_{0}\left(R_{\infty}-R\right) \dot{p}+r_{1}\left(R_{0}-R\right)\left|R_{0}-R\right|^{r_{2}-1}
$$

where $r_{0}, R_{\infty}, r_{1}, R_{0}$, and $r_{2}$ are all parameters. The initial value of the isotropic hardening parameter is zero.

\subsubsection{Kinematic hardening}

The net backstress is the sum of three individual backstress terms:

$$
\boldsymbol{X}=\sum_{i=1}^{3} \boldsymbol{X}_{i}
$$

The evolution equation for each individual backstress is

$$
\dot{\boldsymbol{X}}=\frac{2}{3} c(p, \dot{p}) \dot{\boldsymbol{\varepsilon}}_{v p}-\frac{c\left(p, \dot{p}_{0}\right)}{L(p)} \dot{p} \boldsymbol{b}-\chi(T) x_{0} \Phi^{(x)}(p)\left(\sqrt{\frac{3}{2}} \frac{\|\boldsymbol{X}\|}{D}\right)^{x_{1}} \frac{\boldsymbol{X}}{\|\boldsymbol{X}\|}
$$

where $x_{0}$ and $x_{1}$ are parameters, $c(p, \dot{p})$, is a function defined below of the the equivalent plastic strain, $p$ and either the actual plastic strain $\dot{p}$ or a reference plastic strain rate $\dot{p}_{0}$, $\Phi^{(x)}(p)$ is a softening function with independent parameters,

$$
L(p)=l\left(l_{1}+\left(1-l_{1}\right) \exp \left[-l_{0} p\right]\right)
$$

with $l, l_{0}$, and $l_{1}$ parameter, and

$$
\boldsymbol{b}=\left(1-b_{0}\right) \boldsymbol{X}+\frac{2}{3} b_{0}(\boldsymbol{n} \otimes \boldsymbol{n}): \boldsymbol{X}
$$

with $b_{0}$ a parameter and

$$
\boldsymbol{n}=\sqrt{\frac{3}{2}} \frac{s-\boldsymbol{X}}{\|s-\boldsymbol{X}\|} .
$$

In these expressions the outer product symbol $\otimes$ between two rank two tensors denotes the product given in index notation as

$$
\boldsymbol{a} \otimes \boldsymbol{b}=a_{i j} b_{k l} .
$$


The backstresses all start at $\boldsymbol{X}=\mathbf{0}$.

Walker's original model defined

$$
c(p, \dot{p})=\left\{c_{0}+c_{1} \dot{p}^{1 / c_{2}}\right\} \Omega(p)
$$

with the $\Omega$ function of the equivalent plastic strain given as

$$
\Omega(p)=1+\left(\frac{D-D_{0}}{D_{\xi}}\right)^{\omega_{0}} \omega(p)\left(\omega_{1}-1\right) \exp \left(-\omega_{2} q\right)
$$

with $\omega_{0}, \omega_{1}$, and $\omega_{2}$ parameters. The function $\omega(p)$ is defined as

$$
\omega(p)=\omega_{3}+\left(1-\omega_{3}\right) \exp \left[-\omega_{4} p\right]
$$

with $\omega_{3}$ and $\omega_{4}$ additional parameters and $q$ is an additional internal variable with evolution equation

$$
\dot{q}=\dot{p}-\chi(T) q_{0} q
$$

where $q_{0}$ is a parameter and $q(0)=0$. The function $\Omega(p)$ and the associated internal variable describe the stress overshoot in the cyclic tests, mentioned in Section 2.1.

This implementation omits the overshoot part of the model, leaving

$$
c(\dot{p})=c_{0}+c_{1} \dot{p}^{1 / c_{2}}
$$

with $c_{0}, c_{1}$, and $c_{2}$ parameters. Depending on the location of the $c$ function (in the hardening or dynamic recovery terms), this function is either invoked with the actual plastic strain rate $\dot{p}$ or with some constant rate $\dot{p}_{0}$, which is a model parameter.

The subsequent tables differentiate the parameters for each backstress using superscripted indices.

\subsubsection{Drag stress evolution}

The drag stress evolves as

$$
\dot{D}=d_{0}\left(1-\frac{D-D_{0}}{D_{\xi}}\right) \dot{p}-\chi(T) \Phi^{(D)}(p) d_{1}\left(D-D_{0}\right)^{d_{2}}
$$

where $d_{0}, D_{\xi}, d_{1}$, and $d_{2}$ are parameters, and $\Phi^{(D)}(p)$ is a softening function with independent coefficients.

Section 2.3 provides tables summarizing the complete set of model parameters.

\subsubsection{Modification for a rate-insensitive response}

One challenge in developing a complete inelastic model for a material is that while all the ASME Section III, Division 5 materials are rate dependent at elevated temperatures, typically in the range of normal component operation, they become rate insensitive at lower temperatures. Components may experience excursions into this rate-independent temperature range, for example during plant shutdowns. Modeling these transients then requires a 
model that can transition from a rate dependent response at higher temperatures to a rate independent response at lower temperatures [11].

Walker developed a method for approximating a rate-independent response starting with a viscoplastic model by alternating the definition of the strain rate [12]. His approach works for any viscoplastic model. Consider a model defined by the viscoplastic rate:

$$
\dot{\varepsilon}_{v p}=\dot{p} \boldsymbol{g}
$$

Walker's method modifies the definition of the scalar plastic strain rate:

$$
\dot{\varepsilon}_{v p}^{(m o d)}=\dot{p}_{m o d} \boldsymbol{g}
$$

with

$$
\dot{p}_{\text {mod }}=\kappa \dot{p} .
$$

The modification function is:

$$
\kappa=1-\lambda+\frac{\lambda \sqrt{\frac{2}{3}}\|\dot{\boldsymbol{e}}\|}{\dot{\varepsilon}_{\text {ref }}}
$$

where $\lambda$ is a parameter, $\dot{\varepsilon}_{r e f}$ is a reference strain rate, and is the deviatoric part of the total strain rate $\dot{\boldsymbol{e}}=\operatorname{dev}(\dot{\boldsymbol{\varepsilon}})$. In addition, all terms in the model evolution equations directly proportional to time must also be modified by the $\kappa$ factor. In the Walker model described here this means the factor is applied to the plastic strain rate (Eq. 2.8) as well as to the static recovery terms in the internal variable evolution Eqs. 2.11, 2.13, and 2.22. For example, the backstress evolution equation (Eq. 2.13) becomes

$$
\dot{\boldsymbol{X}}=\frac{2}{3} c(p, \dot{p}) \dot{\boldsymbol{\varepsilon}}_{v p}-\frac{c\left(p, \dot{p}_{0}\right)}{L(p)} \dot{p} \boldsymbol{b}-\kappa \chi(T) x_{0} \Phi^{(x)}(p)\left(\sqrt{\frac{3}{2}} \frac{\|\boldsymbol{X}\|}{D}\right)^{x_{1}} \frac{\boldsymbol{X}}{\|\boldsymbol{X}\|}
$$

where applying the $\kappa$ factor to the equivalent plastic strain rate (Eq. 2.8) takes care of the scaling on the first two terms. The NEML library was already configured to separate out plastic strain, time, and temperature rate expressions and so implementing Walker's modification simply required applying the $\kappa$ factor to the portions of the total rates proportional to plastic strain and time.

One way to think about Walker's modification is as an adjusted time increment [12]. This means the scale factor should be applied to any term that is multiplied by the discrete time increment $\Delta t$ in the numerical integration.

With an appropriate reference strain rate this model gives the base, rate-dependent response for $\lambda=0$ and approximates the rate-independent response for $\lambda \approx 1$. By changing the value of $\lambda$ with temperature the model can transition from a rate-independent response at lower temperatures to a rate-dependent response at higher temperatures. The reference strain rate should be on the same order of magnitude as the applied strain rate. However, numerical experimentation on a simple viscoplastic model shows the response of the modification term is relatively insensitive to the reference strain rate. Any value within a few orders of magnitude of the simulated strain rates seems to produce a rate insensitive response for $\lambda \approx 1$. 


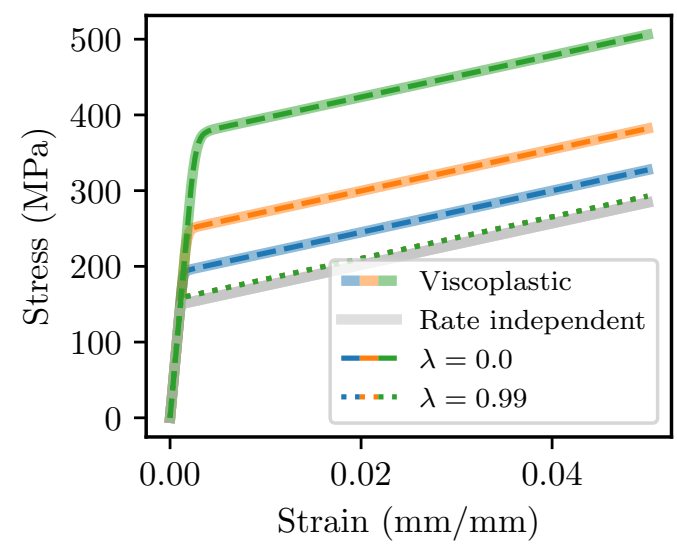

Figure 2.1: Flow curves from four different model implementations at three different strain rates demonstrating the effectiveness of Walker's method for transitioning from a viscoplastic, rate-dependent response to a rate-insensitive response approximating classical rate independent plasticity. The three strain rates applied were $10^{-6}, 10^{-4}$, and $10^{-2} 1 / \mathrm{s}$. The dotted lines giving the $\lambda=0.99$ results all overlap, i.e. this model gives the same response regardless of the applied strain rate.

We implemented this rate-modification algorithm in NEML in a generic fashion so that it can be used with any viscoplastic model. Figure 2.1 shows the effect of decreasing the parameter $\lambda$ for a simple Perzyna viscoplastic model. The figure plots stress/strain curves using four different models at three different values of strain rate. The four models are:

- Classical viscoplasticity (no rate switching)

- Classical rate-independent plasticity

- Walker's rate switching formulation with $\lambda=0$

- Walker's rate switching formulation with $\lambda=0.99$.

This figure validates the rate-switching mechanisms: with $\lambda=0$ the model exactly matches the classical rate-dependent formulation. As $\lambda \rightarrow 1$ the model becomes rate-insensitive and the model response approaches the classical rate-independent formulation.

\subsection{Parameter set identification and verification}

The four parameter sets "A", "B", "C", and "D" were recovered from the ORNL development work. The unit system, as described here, uses megapascals for stress, Kelvin for temperature, and seconds for time. These four parameter sets share the same reference strain rate, temperature, rate-switching parameters, and other basic data described in Tables 2.1 and 2.2 .

Tables 2.3-2.6 then describe the remaining, notionally temperature-dependent material parameters that define each parameter set. 
Initial High Temperature Inelastic Constitutive Model for Alloy 617

August 2020

\begin{tabular}{|l|l|l|}
\hline Parameter & Description & Value \\
\hline \hline$\dot{\varepsilon}_{0}$ & Viscoplastic reference rate & 1.0 \\
\hline$\dot{\varepsilon}_{r e f}$ & Rate-switching reference rate & $1 \mathrm{e}-4$ \\
\hline$T_{r e f}$ & Reference temperature & 1223 \\
\hline$R$ & Gas constant & 8.314472 \\
\hline$\dot{p}_{0}$ & Reference plastic strain rate & $1 \mathrm{e}-2$ \\
\hline
\end{tabular}

Table 2.1: Fixed, temperature-independent parameters for all four model.

While potentially each of the 50 individual parameters in Tables 2.3-2.6 can be temperature dependent, in practice only parameter set "A" has temperature-dependent hardening parameters (and even then, only for the third backstress term). The model has 55 temperature-dependent parameters and 4 fixed parameters.

Unfortunately, verifying the NEML implementation of the Walker model is difficult. Error could arise in two ways:

- The implementation of the mathematical model description in Section 2.2 could be incorrect. The potential for error here is slight as the references cited above describe the Walker model in exact detail and so the equations implemented in NEML could be verified by direct comparison to the equations developed in the ORNL work.

- The model constants described in Tables 2.1-2.6 could be entered incorrectly into the NEML input system. There are two sub-possibilities:

- Parameters in Walker's original derivation and implementation were renamed several times. The tables above could swap the definition of two parameters. This possibility is low as this is likely to produce a radically incorrect response in the model.

- Parameters could be mistranscribed from the original source material (here, the Fortran UMAT implementation). This possibility is quite likely given the large number of parameters.

The best way to verify the NEML implementation would be to compare to a simulation result published in the original source material. Unfortunately the original reports do not show many simulation results. In fact, the report describing the four parameter sets reconstructed here does not show a comparison between all four instantiations of the model for any conditions. The closest the report comes is showing results for "A" and "C" parameter sets for a SMT test performed at ORNL [13]. Regrettably, the report does not provide the information needed to reconstruct the test conditions in a simulation.

From Walker's exposition we know the model was primarily calibrated against the INL strain controlled cyclic tests at $950^{\circ} \mathrm{C}$. Based on this information, we surmise all four variants should perform reasonably well when compared to this dataset, which the authors have access to. Figure 2.2 compares the four parameters sets to one of these tests: a fully reversed, straincontrolled cyclic test with a total strain range of $1 \%$ and a 10 minute hold. The plots compare the INL data to the model results for the four parameter sets for the first 10 cycles. 
Initial High Temperature Inelastic Constitutive Model for Alloy 617

August 2020

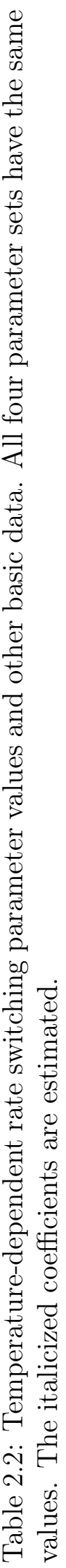


Initial High Temperature Inelastic Constitutive Model for Alloy 617

August 2020

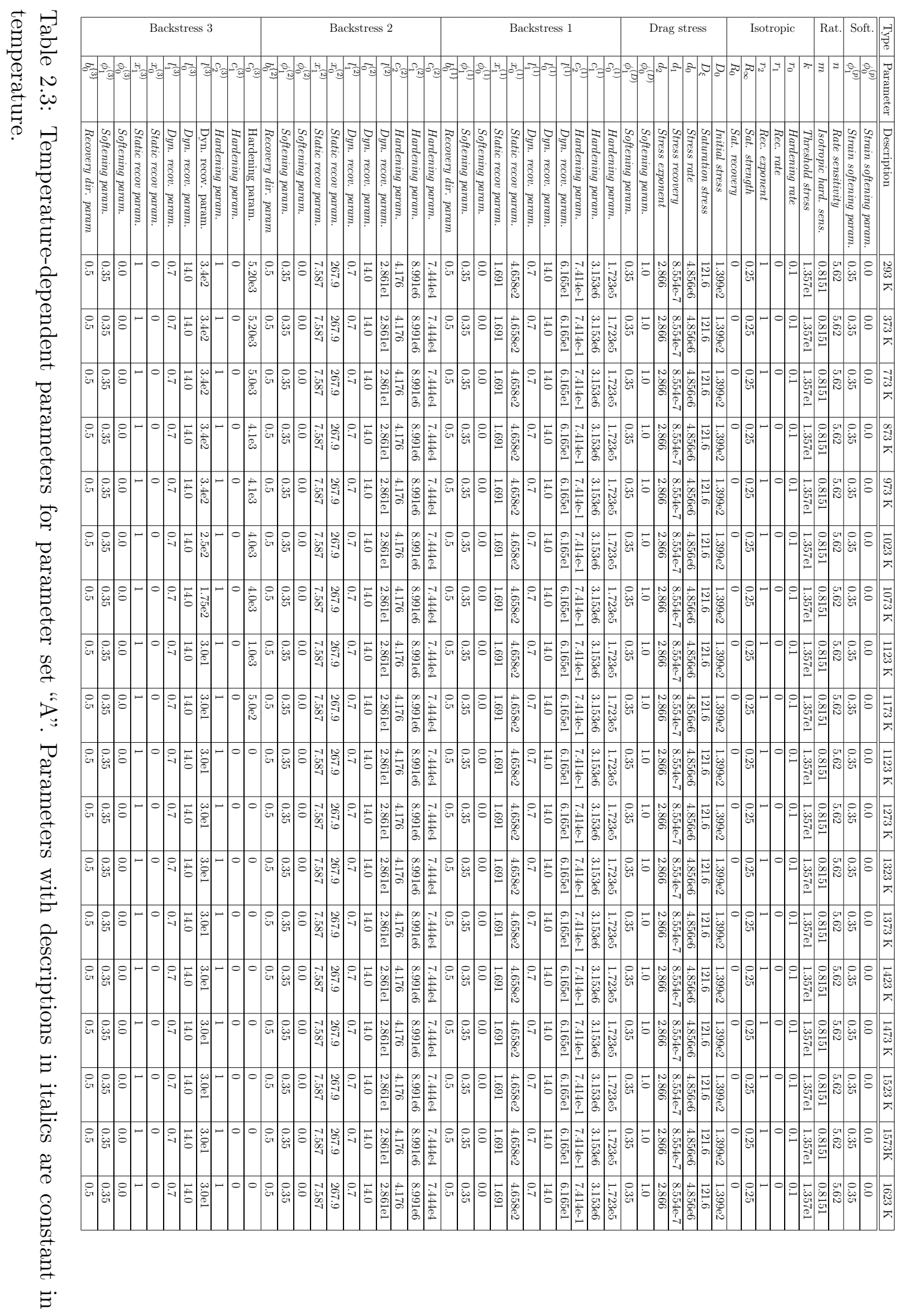


Initial High Temperature Inelastic Constitutive Model for Alloy 617

August 2020

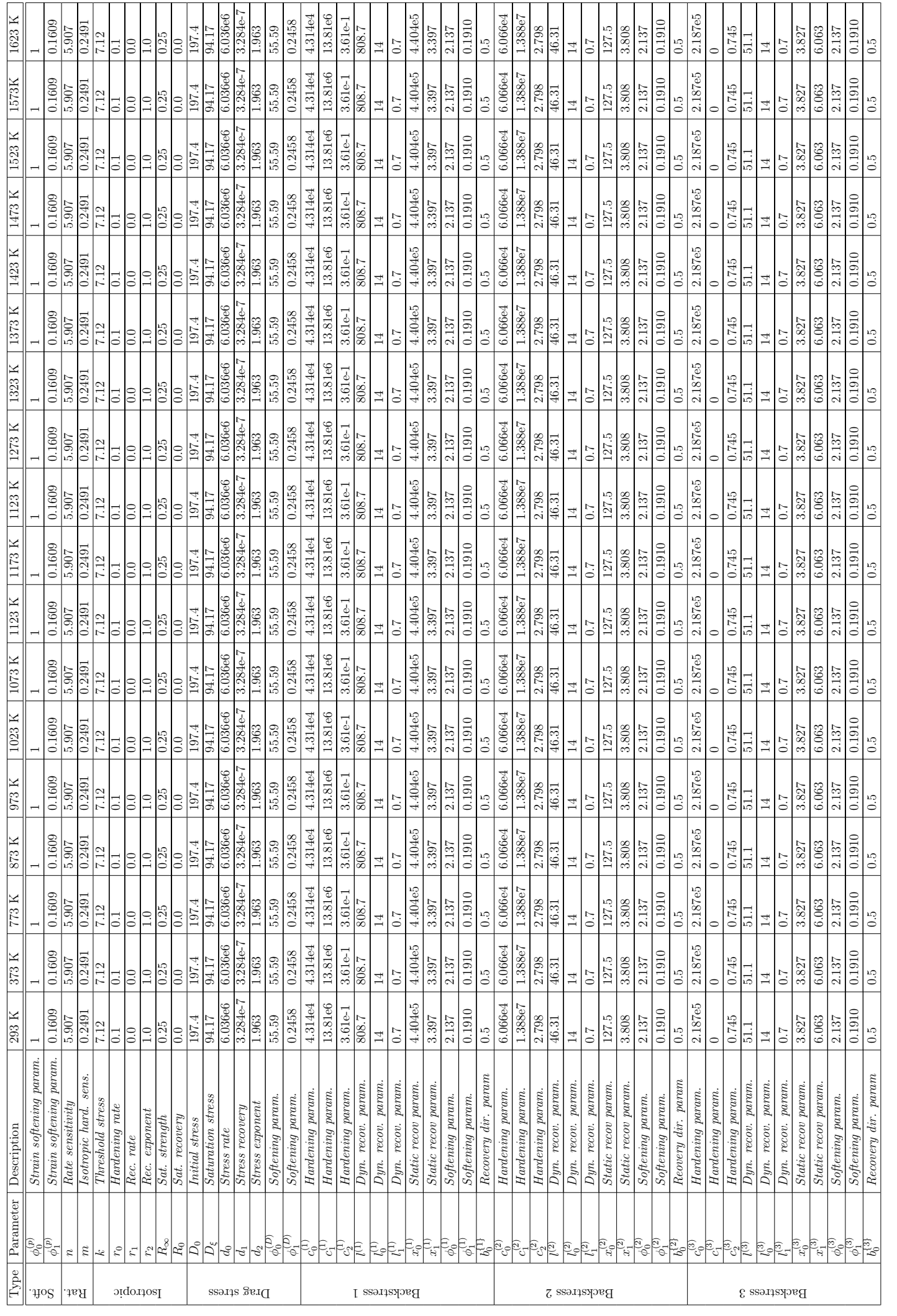

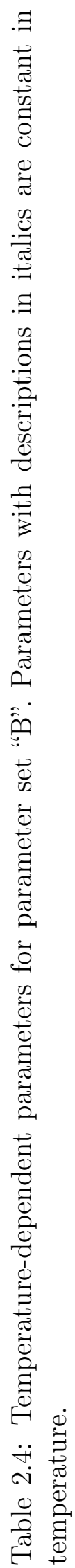


Initial High Temperature Inelastic Constitutive Model for Alloy 617

August 2020

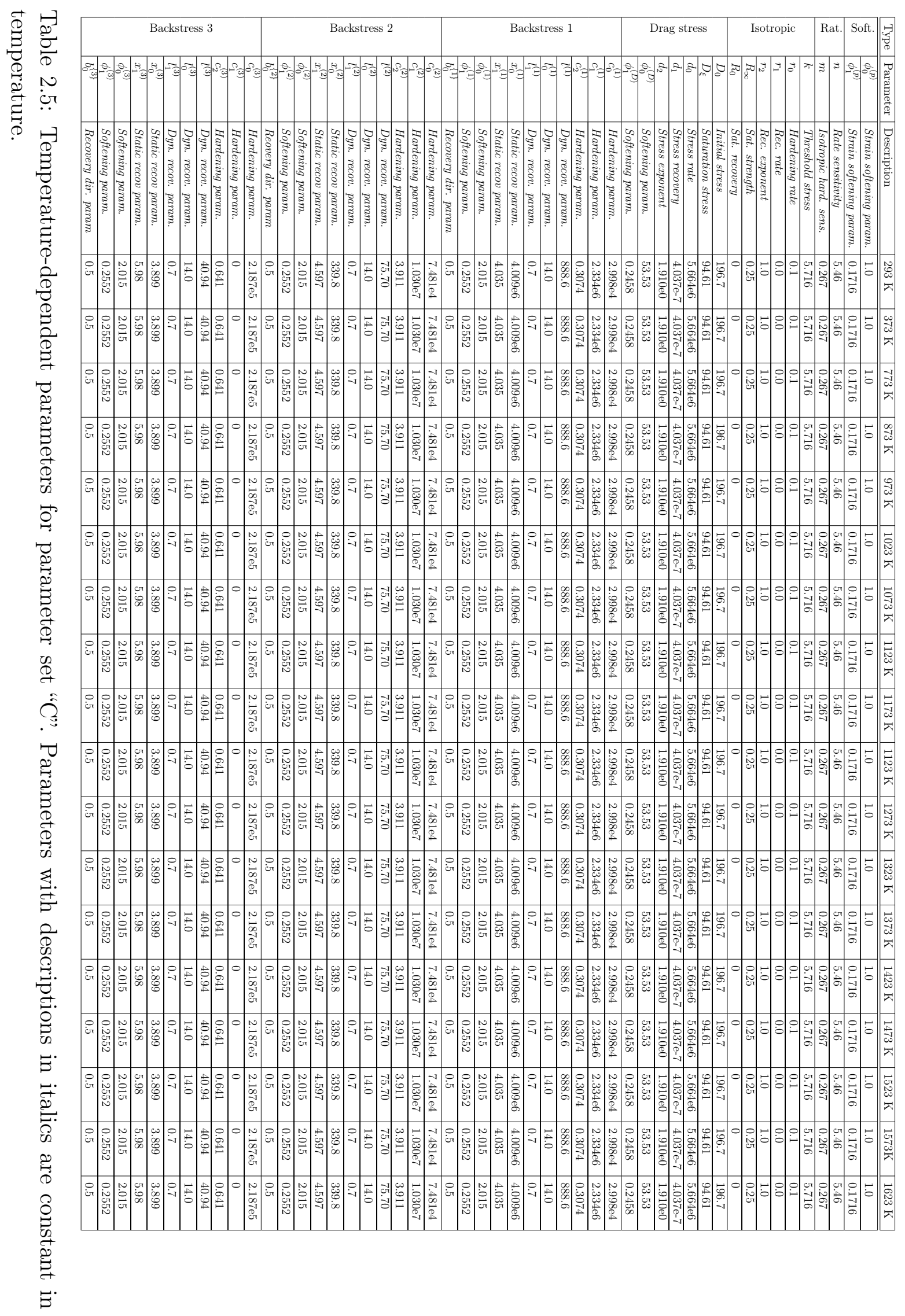


Initial High Temperature Inelastic Constitutive Model for Alloy 617

August 2020

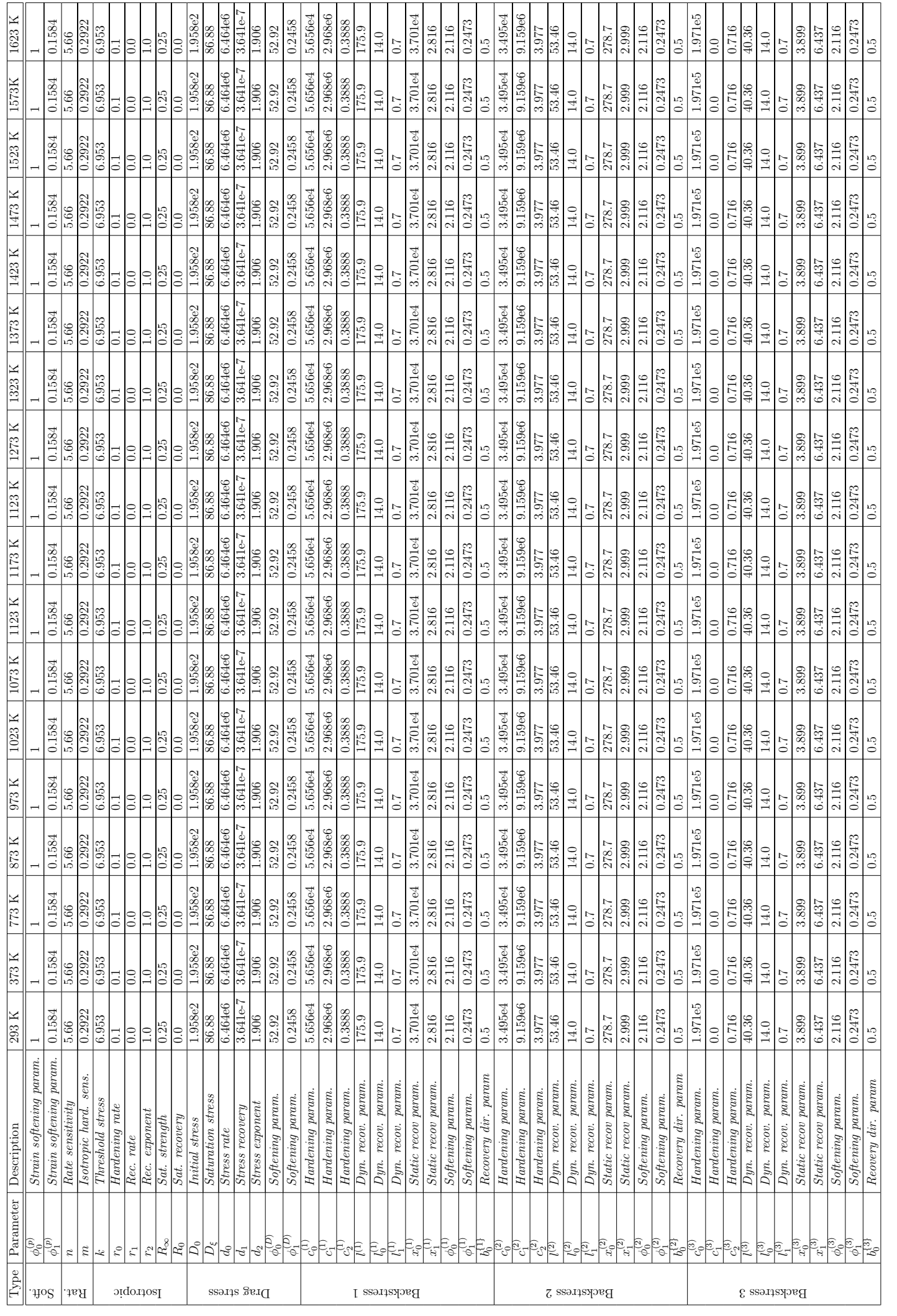

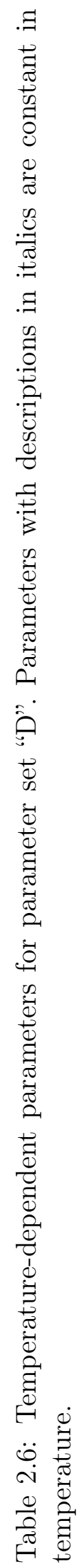




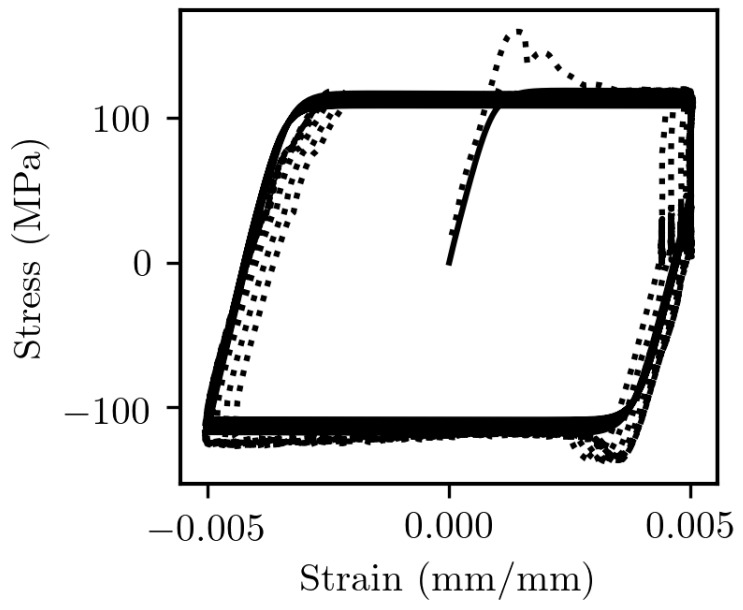

(a) Parameter set "A".

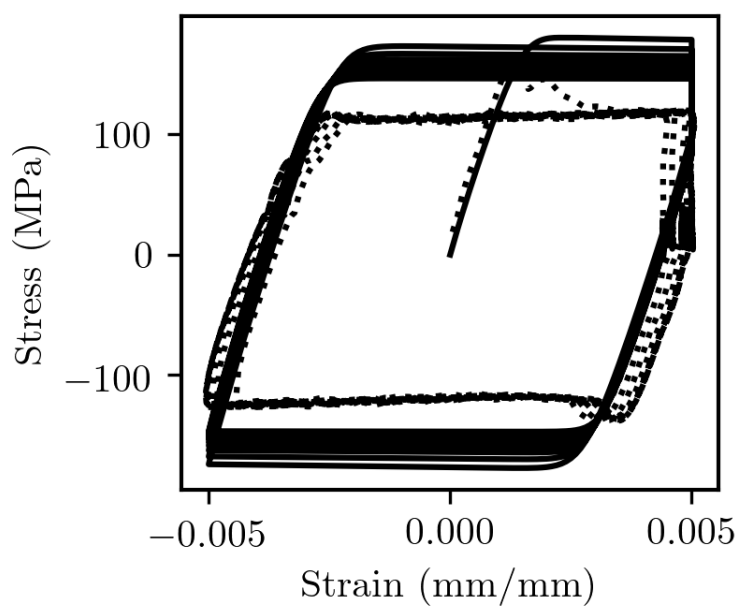

(c) Parameter set "C".

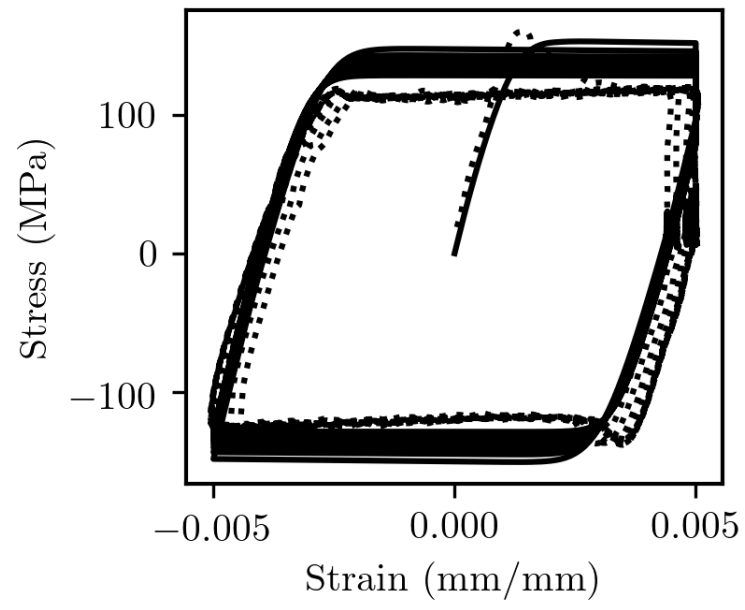

(b) Parameter set "B".

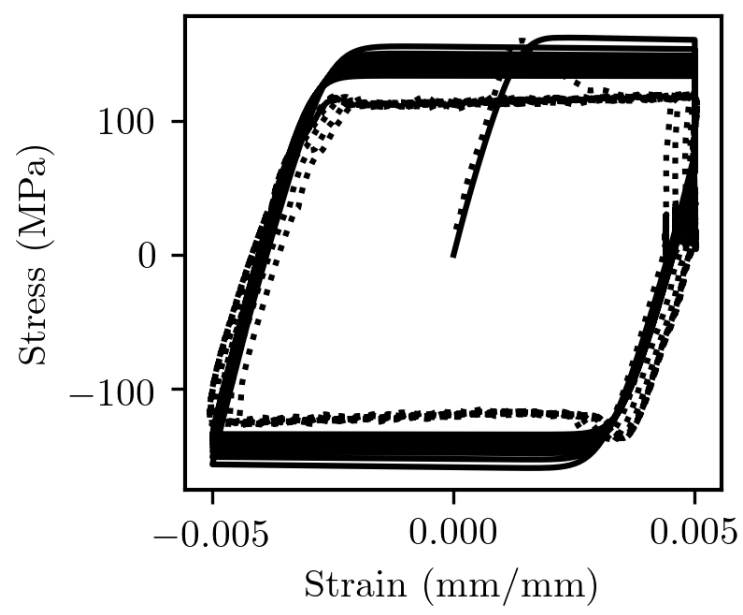

(d) Parameter set "D".

Figure 2.2: Comparison between the model results and a fully-reversed strain-controlled cyclic experiment at $950^{\circ} \mathrm{C}$ for a total strain range of $1 \%$ and a 10 minute hold time. The solid lines are the model results, the dashed lines are experiments. 
The comparison shows that all four parameter sets reasonably describe the experimental data. This implies the NEML model likely faithfully implement's Walker's model. Unfortunately this verification exercise does not prove the $50+$ constants in the model were correctly transcribed, but it at least demonstrates there are no gross errors in the implementation. The four parameter sets bracket the INL experiments. Parameter set "A" accurately hits the initial flow stress neglecting the overshoot (which the NEML implementation does not attempt to capture with Walker's overshoot model), the other parameter sets have an initial flow stress between the initial stable and the initial overshoot values. 



\section{Model assessment}

\subsection{Comparison to data}

This chapter compares the response of the Walker model to the available Alloy 617 experimental data. For this most part, this data was collected at INL as part of the NGNP program and the Alloy 617 ASME Code Case effort. A few additional tests were collected from the literature, notably from references [14] and [15]. This dataset includes tension tests, creep tests, and strain controlled cyclic tests with and without holds (i.e. fatigue and creep-fatigue tests). Stress relaxation data was extracted from the creep-fatigue tests by separating the stress relaxation portion of the first cycle into a separate data set.

\subsubsection{Rate sensitivity via Kocks-Mecking diagrams}

As mentioned in Chapter 1, an inelastic model used in advanced reactor design may need to accurately capture the material rate sensitivity across a wide variety of temperatures and strain rates. Kocks-Mecking diagrams are a method for summarizing material rate sensitivity as a function of strain rate and temperature $[16,17]$. These diagrams plot the material flow stress, normalized by the shear modulus, against a normalized activation energy calculated as a function of the deformation strain rate and temperature. These diagrams can be generated by collating uniaxial tension, creep, and stress relaxation data, as described by [11].

Figure 3.1 plots a Kocks-Mecking diagram for A617 generated from the available creep, tension, and stress relaxation data. The figure overlays the experimental data with a model for material rate senstivity for Alloy 617 calibrated to this data [11]. This model predicts a transition from a rate sensitive behavior to a rate insensitive behavior in A617 at a critical combination of strain rate and temperature. The figure also plots points from simulated tension tests at various temperatures and strain rates using the four parameter sets for the Walker model.

All four parameter sets reasonably predict the material flow stress and the transition between a rate sensitive and rate insensitive response except for a cluster of points associated with the fast strain rates at elevated temperature. This is because the Walker model assumes rate sensitivity only changes as a function of temperature, whereas a model based on the Kocks-Mecking theory posits that inelasticity also becomes rate insensitive for very fast strain rates. The difference is not significant for models intended for reactor design as reactor components are unlikely to experience strain rates fast enough to induce rate insensitive flow, except perhaps for extreme accident conditions.

Between the four parameter sets, the "A" set seems to most accurately represent the material flow stress across a broad range of temperatures and strain rates.

\subsubsection{Flow curves}

INL completed a series of tensile tests at temperatures spanning from room temperature to $1000^{\circ} \mathrm{C}$ at the standard ASTM strain rate of $8.33 \times 10^{-5} \mathrm{~mm} / \mathrm{mm} / \mathrm{s}$. This data set includes replicate tests at all temperatures. Figure 3.2 compares the four parameter sets against the INL tensile curves. 


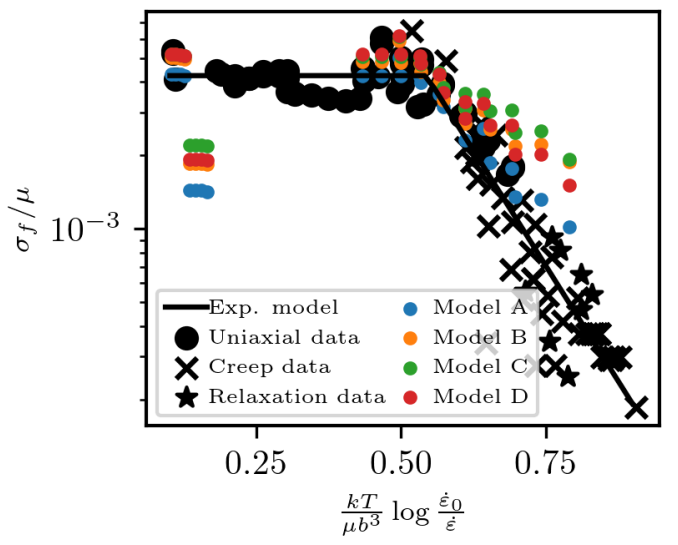

Figure 3.1: Kocks-Mecking diagram for Alloy 617 comparing the experimental data to the Walker model for all four parameter sets.

All four model variants capture the flow curves at high temperature. However, only parameter set "A" captures the lower temperature flow data by including a temperaturedependent work hardening response. The third backstress component in parameter set "A" accounts for this difference. In sets "B", "C", and "D" the third backstress is temperatureindependent and seems to attempt to improve the model cyclic response at the calibration temperature of $950^{\circ} \mathrm{C}$. However, parameter set "A" has a temperature-dependent third backstess clearly aimed at capturing the low temperature work hardening observed in the flow curves.

This backstress term cannot completely capture the shape of the flow curves at low temperature - even parameter set "A" overestimates the amount of dynamic recovery in the flow stress at room temperature. Moreover, parameter set "A" does not correctly capture the intermediate temperature flow curves. All four models are reasonably accurate at higher temperatures, but again parameter set " $\mathrm{A}$ " is somewhat more accurate than the other sets of parameters.

\subsubsection{Creep data}

The experimental data includes creep deformation data from creep tests instrumented with a strain gauge. This data can be plotted either as diagrams showing the total accumulated strain as a function of time at fixed stress and temperature or as plots of creep strain rate versus time. Figures 3.3-3.6 compare the test data against corresponding model predictions using the latter method. These plots show creep rate versus time on a log-log scale. While these plots have several disadvantages - notably the noise in the experimental extensometer data - they have the advantage of showing a broad range of conditions (stresses, rates, and times) on a single plot. The figures show all the available creep test data at four temperatures: $750^{\circ}, 800^{\circ}, 900^{\circ}$, and $1000^{\circ} \mathrm{C}$ with each subfigure showing all test conditions, i.e. stresses, at a given temperature. The figures show both the experimental data and the model prediction. The four figures cover the four parameter sets.

All variants of the Walker model tend to underestimate the initial primary creep rate in 


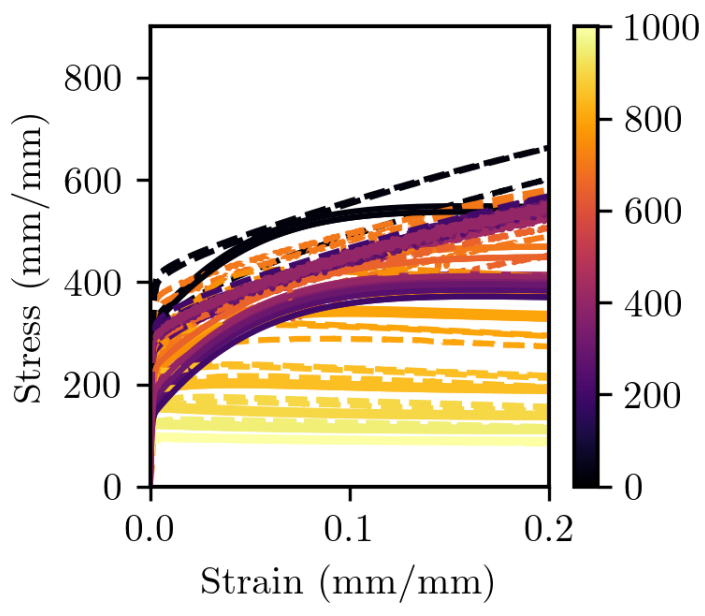

(a) Parameter set "A".

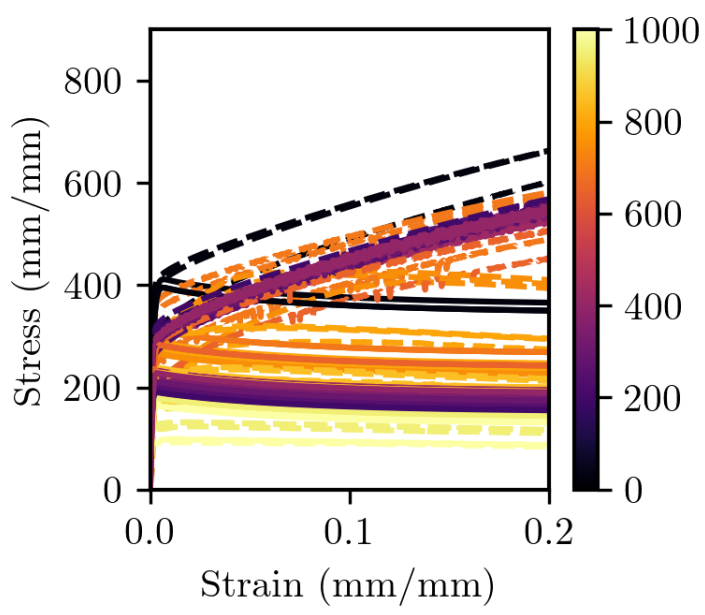

(c) Parameter set "C".

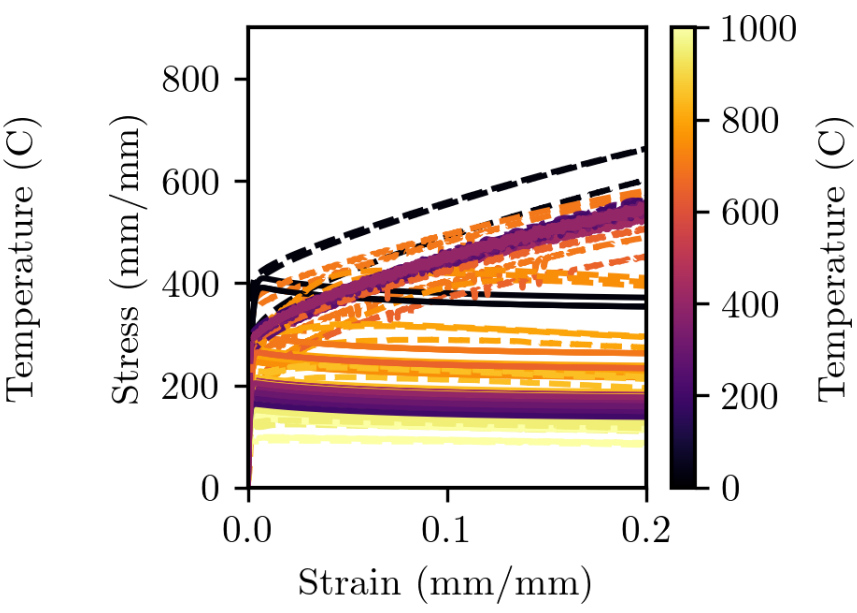

(b) Parameter set "B".

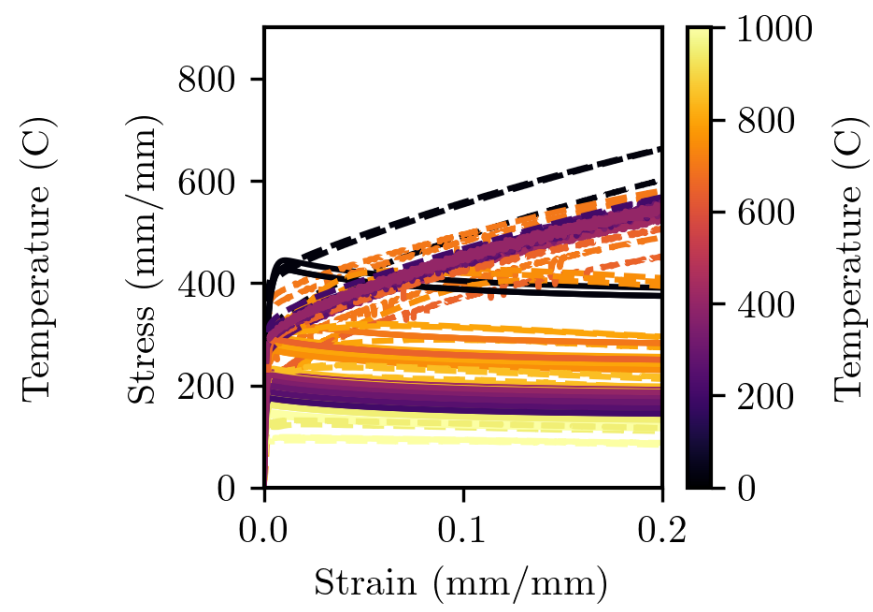

(d) Parameter set "D".

Figure 3.2: Comparison between the model predictions and a collection of uniaxial tension curves collected from INL experimental data. Solid lines are model predictions, dashed lines are corresponding experimental data. The line colors correspond to the test temperature. 


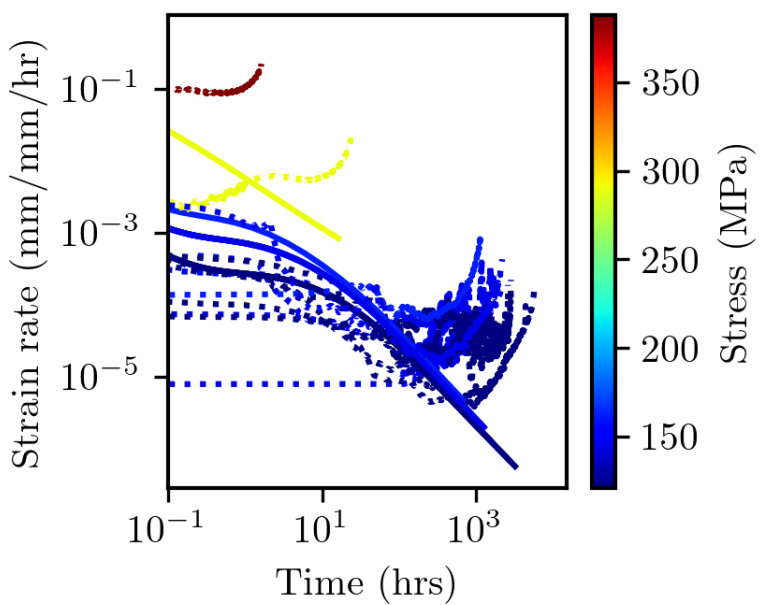

(a) $750^{\circ} \mathrm{C}$

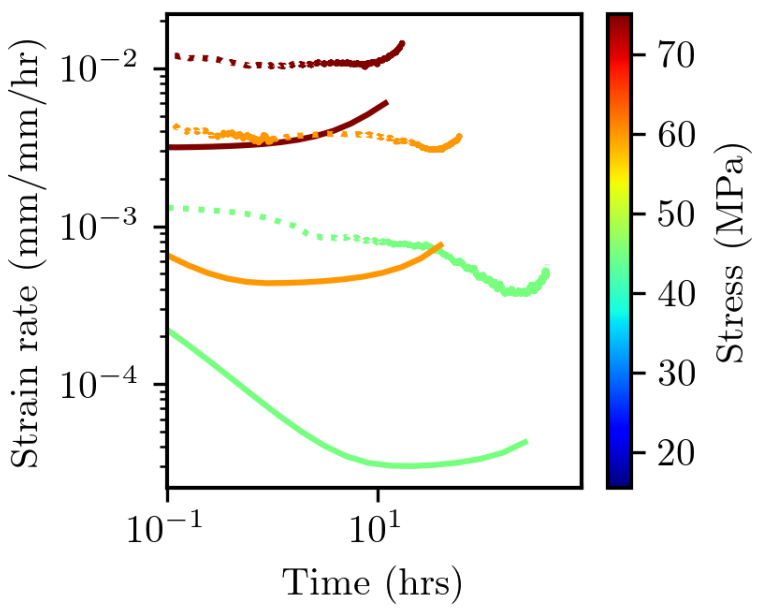

(c) $900^{\circ} \mathrm{C}$

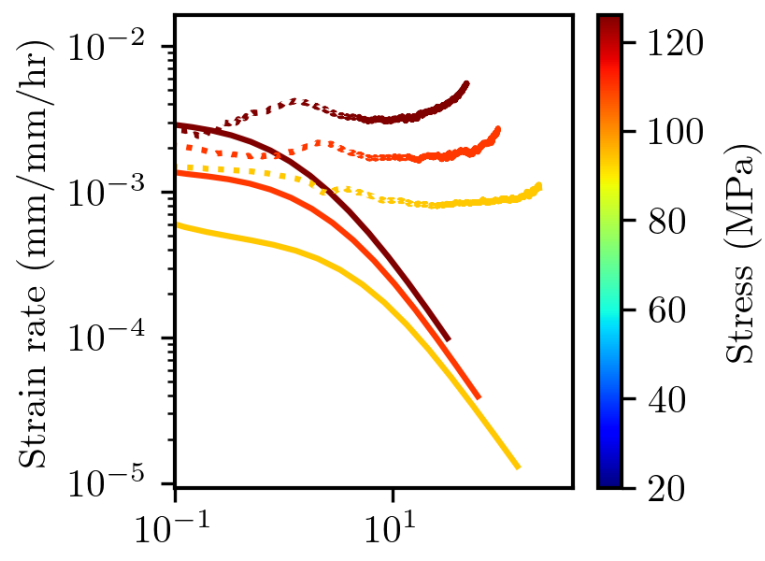

Time (hrs)

(b) $800^{\circ} \mathrm{C}$

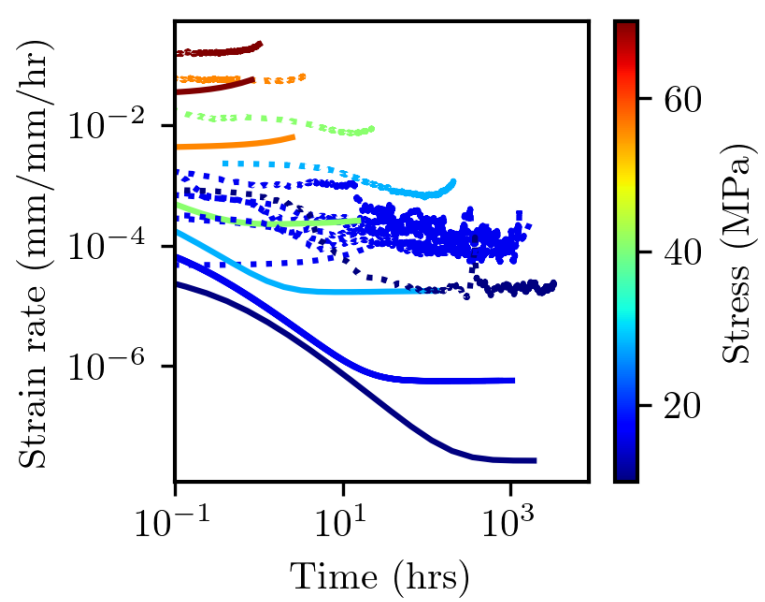

(d) $1000^{\circ} \mathrm{C}$

Figure 3.3: Comparison between parameter set "A" and the creep test data. 


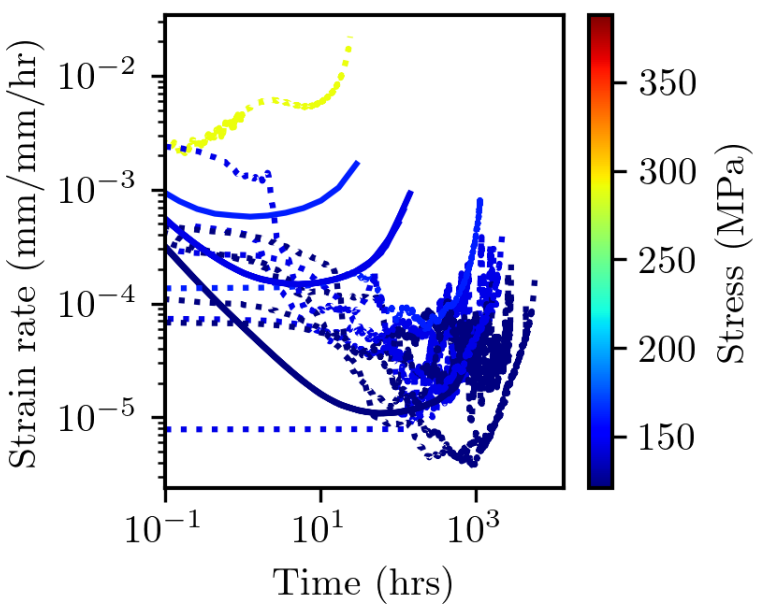

(a) $750^{\circ} \mathrm{C}$

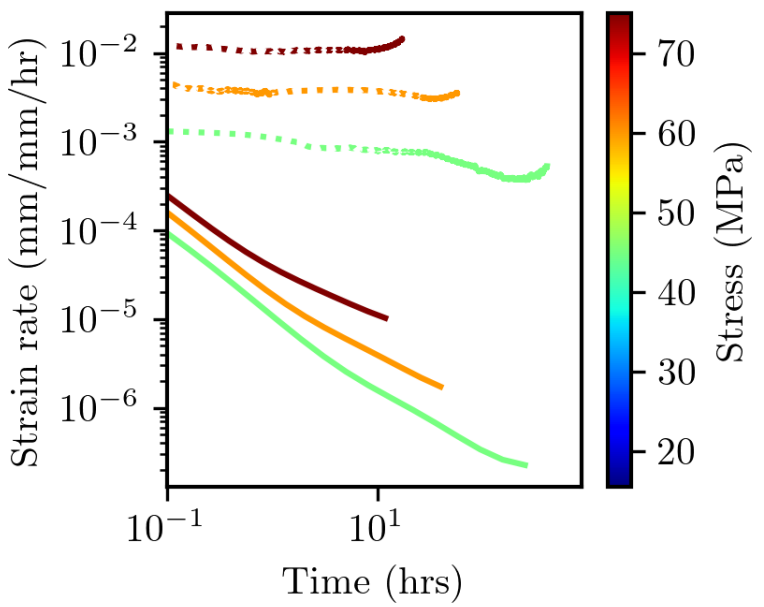

(c) $900^{\circ} \mathrm{C}$

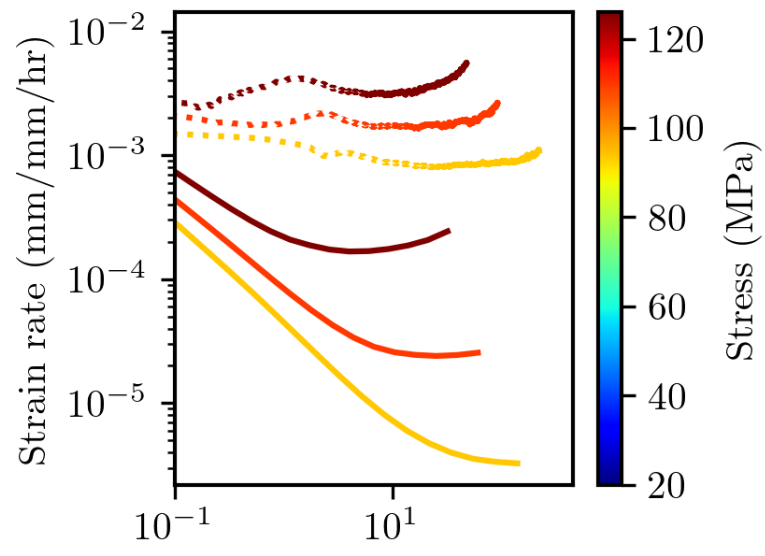

Time (hrs)

(b) $800^{\circ} \mathrm{C}$

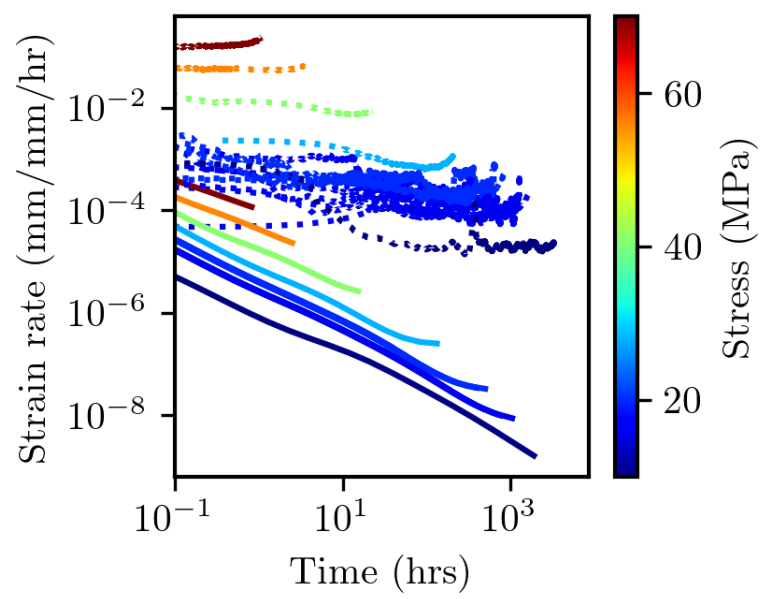

(d) $1000^{\circ} \mathrm{C}$

Figure 3.4: Comparison between parameter set "B" and the creep test data. 


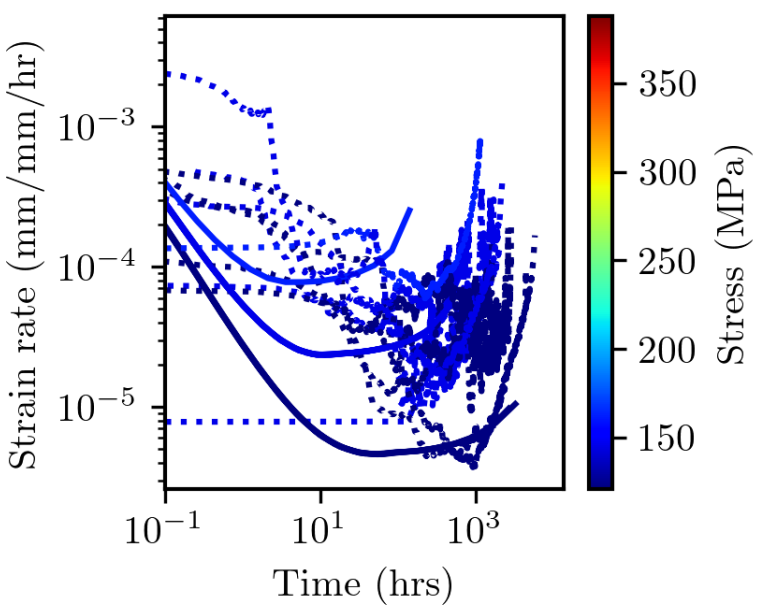

(a) $750^{\circ} \mathrm{C}$

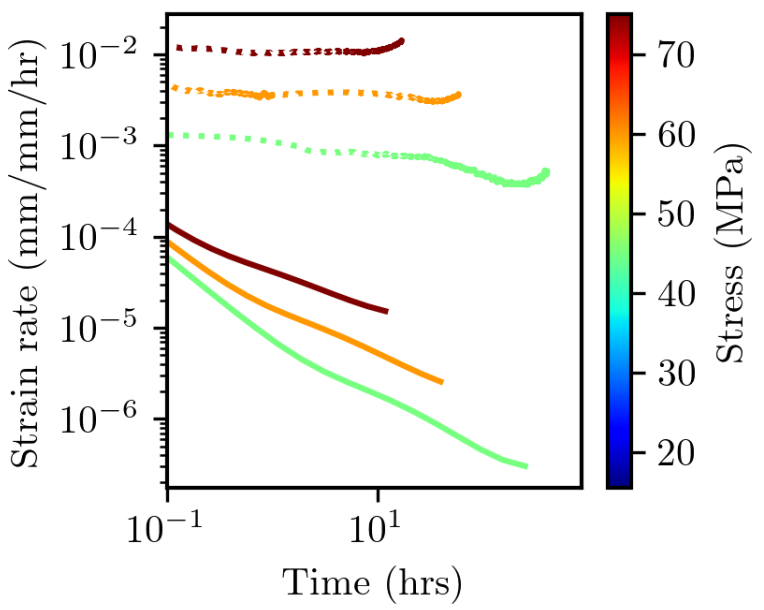

(c) $900^{\circ} \mathrm{C}$

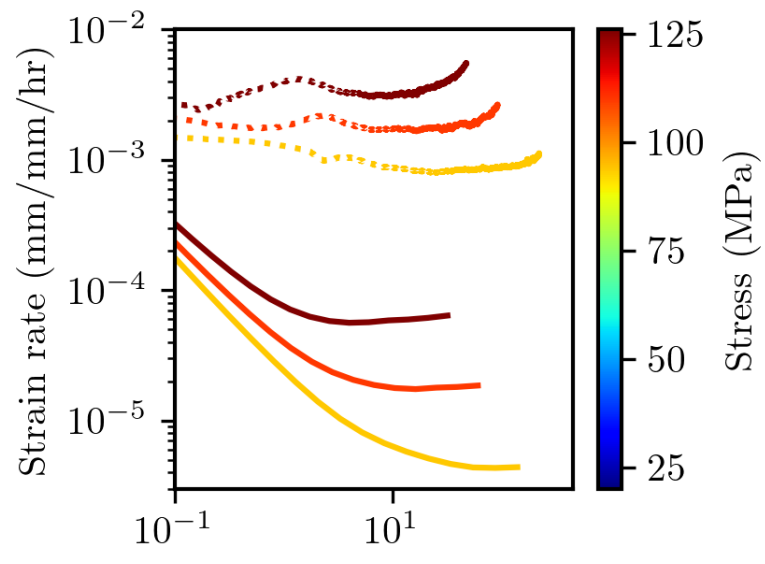

Time (hrs)

(b) $800^{\circ} \mathrm{C}$

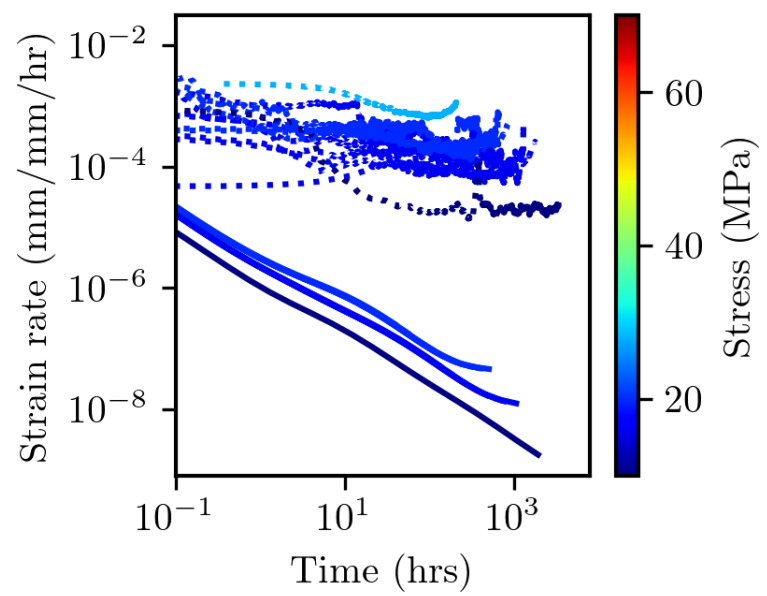

(d) $1000^{\circ} \mathrm{C}$

Figure 3.5: Comparison between parameter set "C" and the creep test data. 


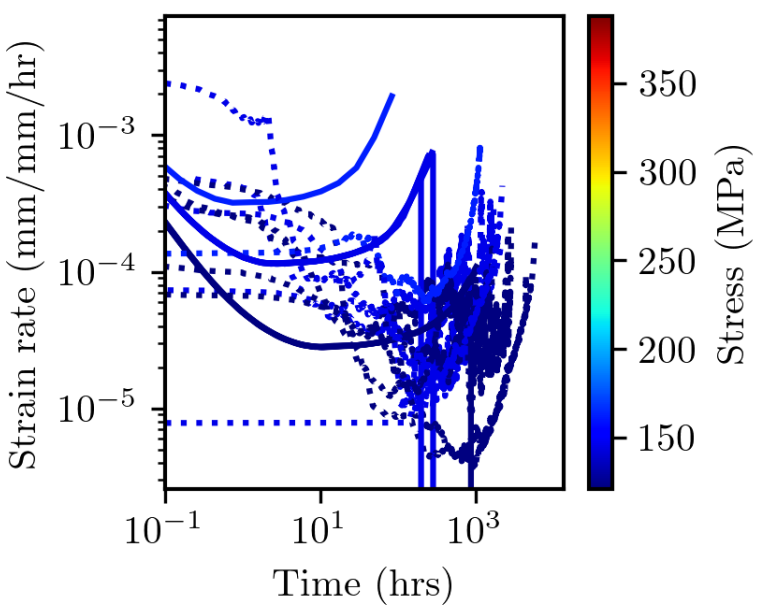

(a) $750^{\circ} \mathrm{C}$

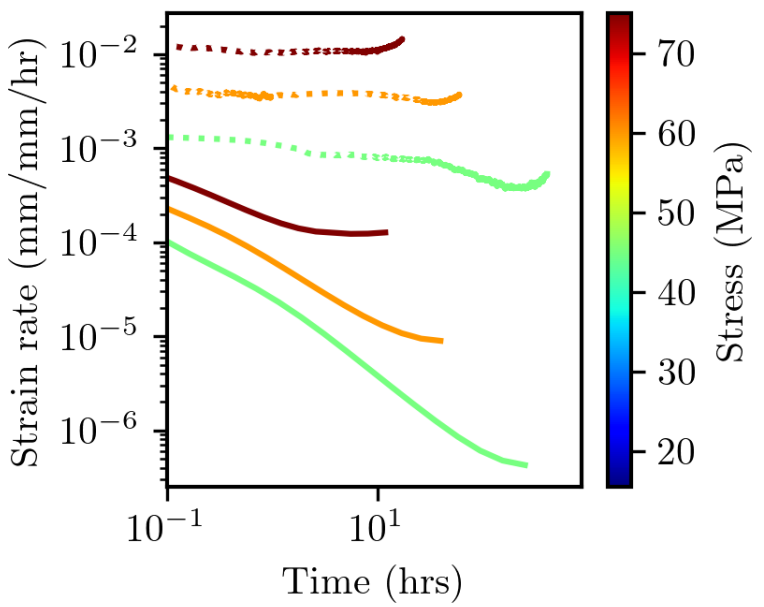

(c) $900^{\circ} \mathrm{C}$

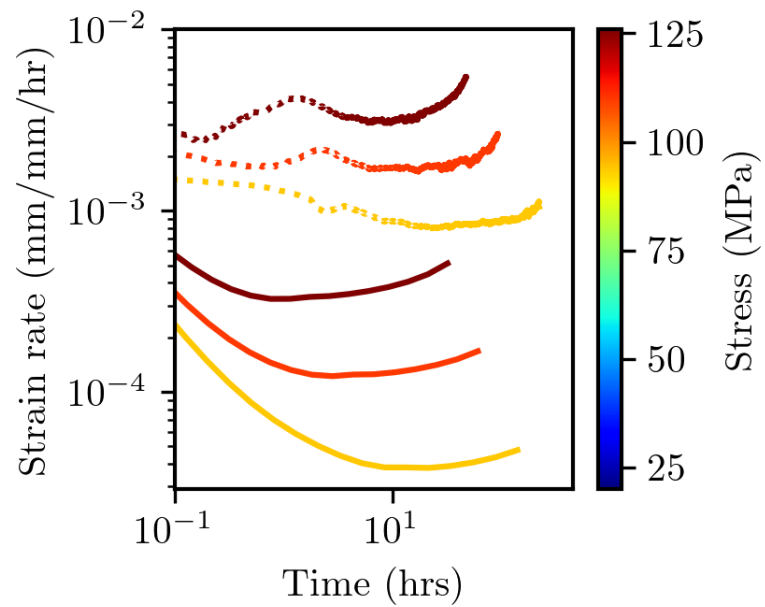

(b) $800^{\circ} \mathrm{C}$

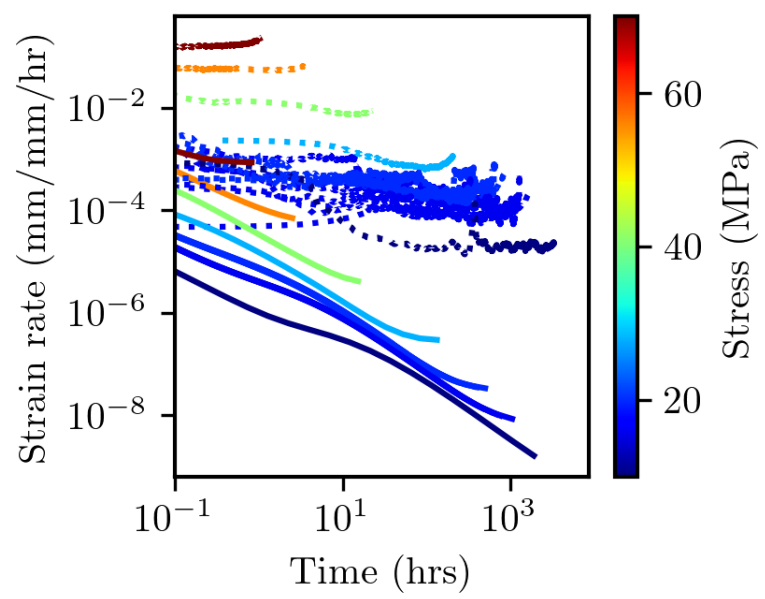

(d) $1000^{\circ} \mathrm{C}$

Figure 3.6: Comparison between parameter set "D" and the creep test data. 


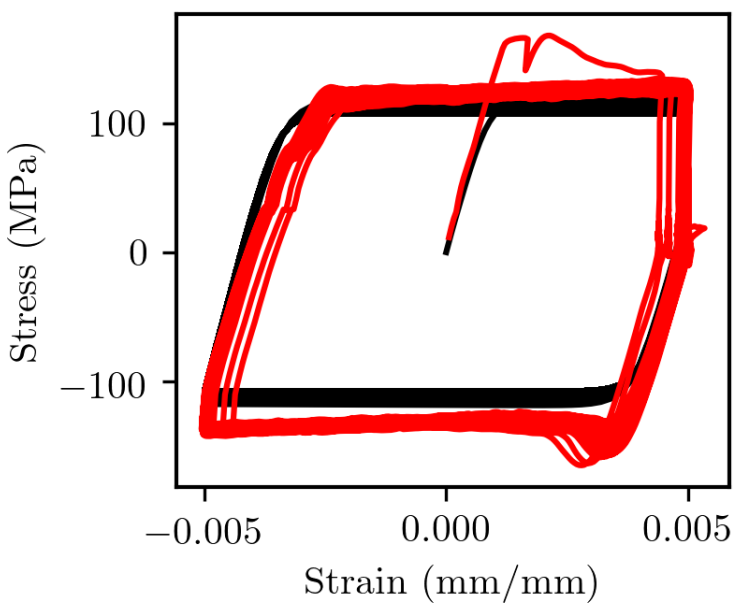

(a) Stress/strain

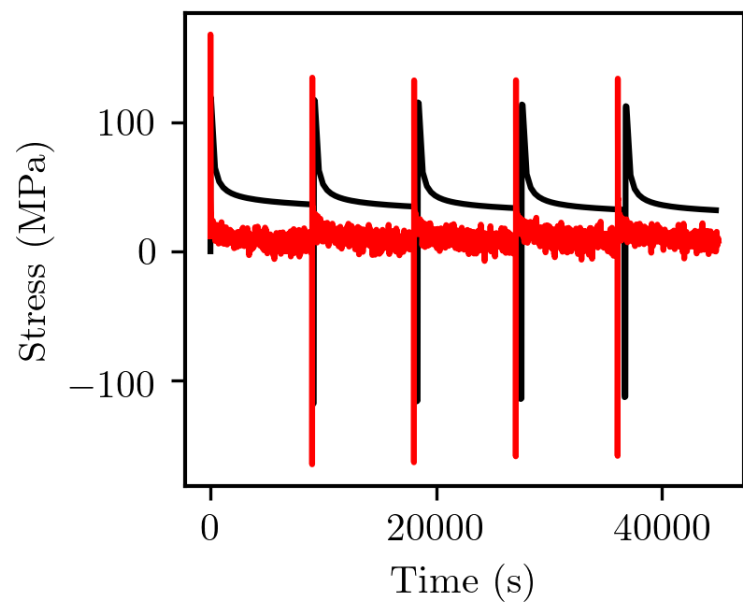

(b) Stress/time

Figure 3.7: Detailed comparison between model results for parameter set "A" (black) and the reference creep-fatigue test (red). Subfigure (a) shows the comparison between the stress/strain hysteresis loops and subfigure (b) shows the comparison as a plot of stress versus time. (a) shows the data for all 100 cycles, (b) shows a truncated time series to highlight the stress relaxation profile over the first few cycles.

Alloy 617, compared to the creep test data. In particular at higher temperatures the Walker model underestimates the creep rates by nearly an order of magnitude. The Walker model has features designed to capture decreasing primary creep rates (via hardening) and a transition to tertiary creep (static recovery). However, except at a few combinations of temperature and stress, these hardening and softening models do not seem to be calibrated to capture the actual transition in A617 between a decreasing and increasing creep rate. Overall, all variants of the model perform poorly relative to the experimental data. Parameter set "A" does the best job of capturing the initial rate of primary creep, whereas the other three parameter sets do a better job of capturing the transition from primary to tertiary creep.

\subsubsection{Strain-controlled cyclic tests}

The Walker model was predominantly calibrated against the INL $950^{\circ} \mathrm{C}$ strain-controlled cyclic test data. All four variants of the model then compare very well against this test data. Generating plots showing a qualitative comparison to these types of experiments is difficult. The full data is a collection of (time,strain,stress) points and for creep-fatigue tests with holds plotting the strain-stress hysteresis loops alone does not give a complete picture of the stress-relaxation portion of the test. One solution is to plot both strain-stress and time-stress diagrams for each test. Figures 3.7-3.10 show this type of comparison for each parameter set for a single creep-fatigue condition $\left(950^{\circ} \mathrm{C}\right.$, fully reversed loading, $1 \%$ total strain range, 2.5 hour tensile hold).

An alternative, condensed approach is to just plot the maximum and minimum stress achieved during a cycle as a function of cycle count. These types of plots abstract much of 


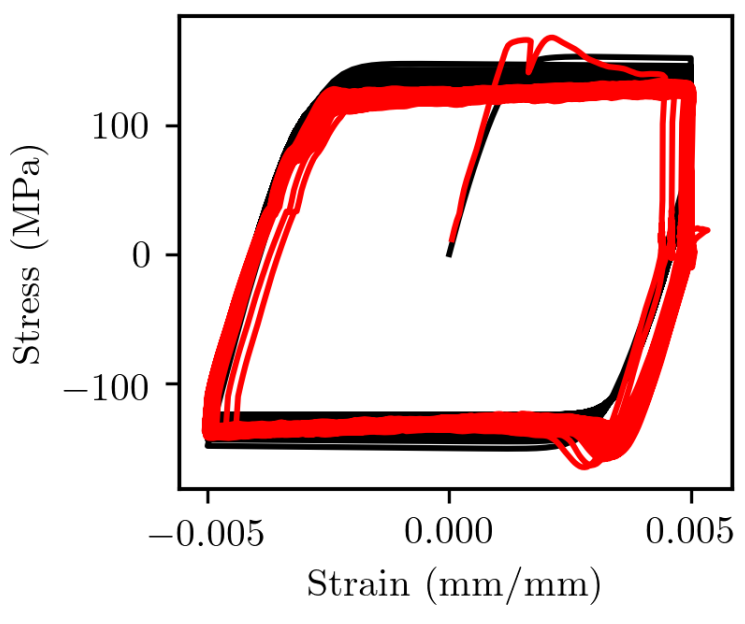

(a) Stress/strain

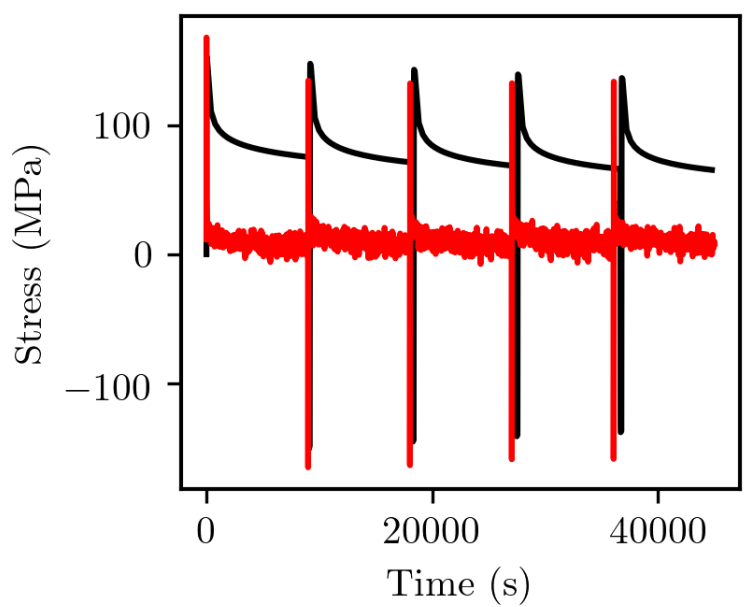

(b) Stress/time

Figure 3.8: Detailed comparison between model results for parameter set "B" (black) and the reference creep-fatigue test (red). Subfigure (a) shows the comparison between the stress/strain hysteresis loops and subfigure (b) shows the comparison as a plot of stress versus time. (a) shows the data for all 100 cycles, (b) shows a truncated time series to highlight the stress relaxation profile over the first few cycles.

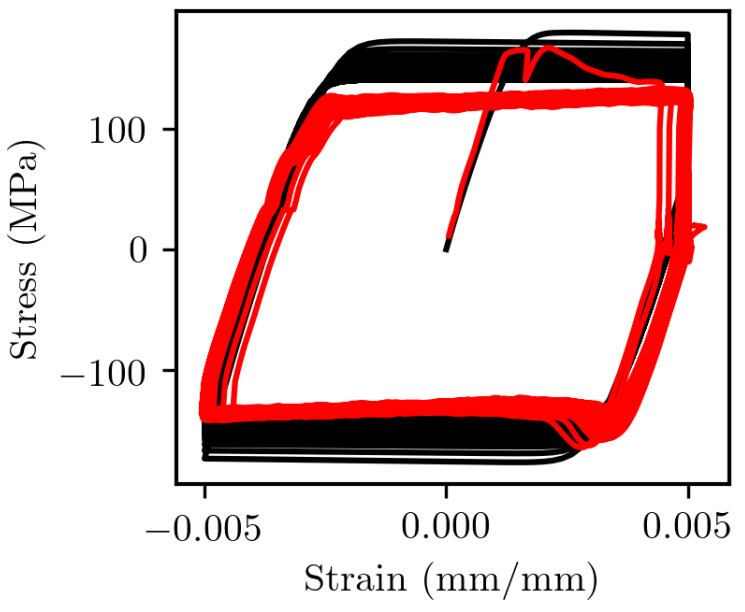

(a) Stress/strain

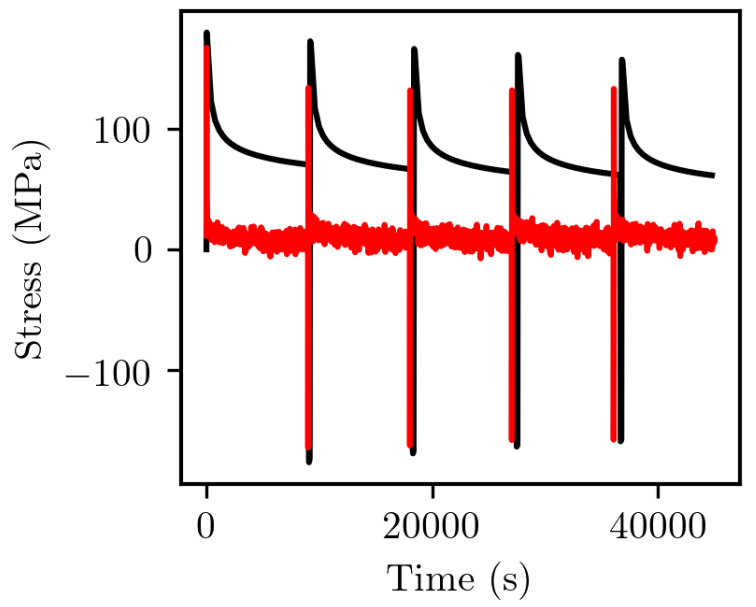

(b) Stress/time

Figure 3.9: Detailed comparison between model results for parameter set "C" (black) and the reference creep-fatigue test (red). Subfigure (a) shows the comparison between the stress/strain hysteresis loops and subfigure (b) shows the comparison as a plot of stress versus time. (a) shows the data for all 100 cycles, (b) shows a truncated time series to highlight the stress relaxation profile over the first few cycles. 


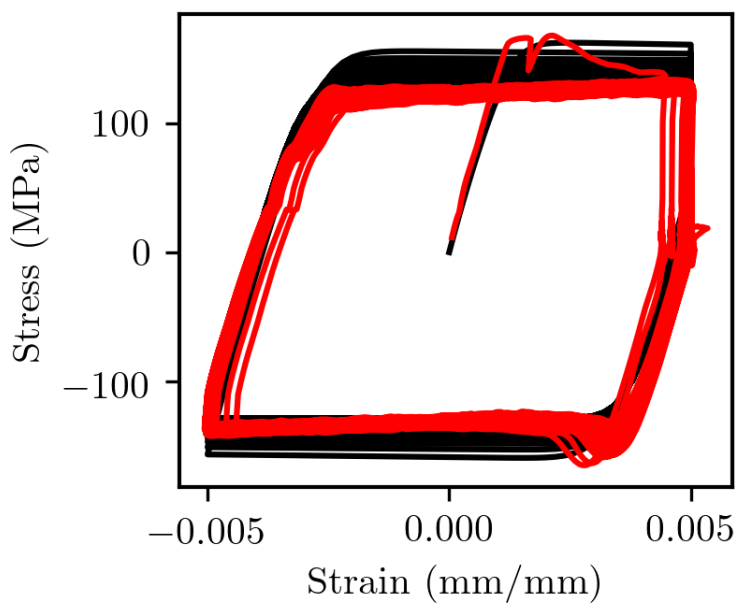

(a) Stress/strain

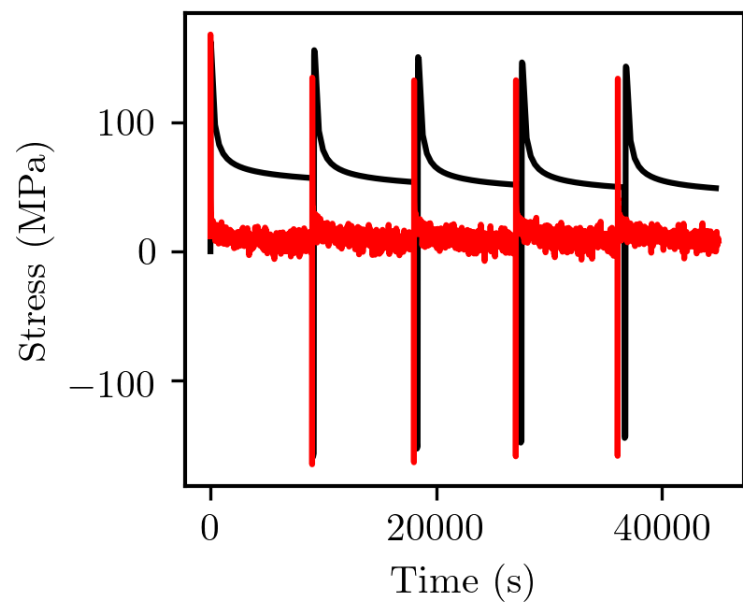

(b) Stress/time

Figure 3.10: Detailed comparison between model results for parameter set "C" (black) and the reference creep-fatigue test (red). Subfigure (a) shows the comparison between the stress/strain hysteresis loops and subfigure (b) shows the comparison as a plot of stress versus time. (a) shows the data for all 100 cycles, (b) shows a truncated time series to highlight the stress relaxation profile over the first few cycles.

the detailed time- and strain-dependent information generated during the test, but have the advantage of being able to summarize a large number of test conditions relatively quickly. Figure 3.11 plots a comparison of this type, showing results from three different test conditions on a single plot (given in the figure legend), again for each parameter set. These comparisons were curtailed at 100 cycle to reduce the time required to run the model simulations.

Finally, a simple, qualitative assessment is to run a simulation corresponding to each test, calculate the average mean relative error in the time/strain/stress history, and sum these results for all the tests for each model. Figure 3.12 is a bar graph comparing the four parameters sets against all the available cyclic data. This plot uses the error measure

$$
R=\frac{1}{N_{\text {test }}} \sum_{N_{\text {test }}} \frac{\int_{t}\left(\frac{\sigma_{\text {exp }}(t)-\sigma_{\text {model }}(t)}{\sigma_{\text {exp }}(t)}\right) d t}{\int_{t} d t}
$$

i.e. the time-averaged relative error summed over all the test conditions.

All four parameter sets reasonably capture the cyclic test data. Again, parameter set "A" is the most accurate. For example, this parameter set better captures the stress relaxation profile in the full comparison and has the lowest relative error.

\subsection{Assessment}

Overall, the Walker model accurately captures the $950^{\circ} \mathrm{C}$ strain-controlled cyclic test data. All four parameter sets perform reasonably compared to this dataset, with "A" having the 


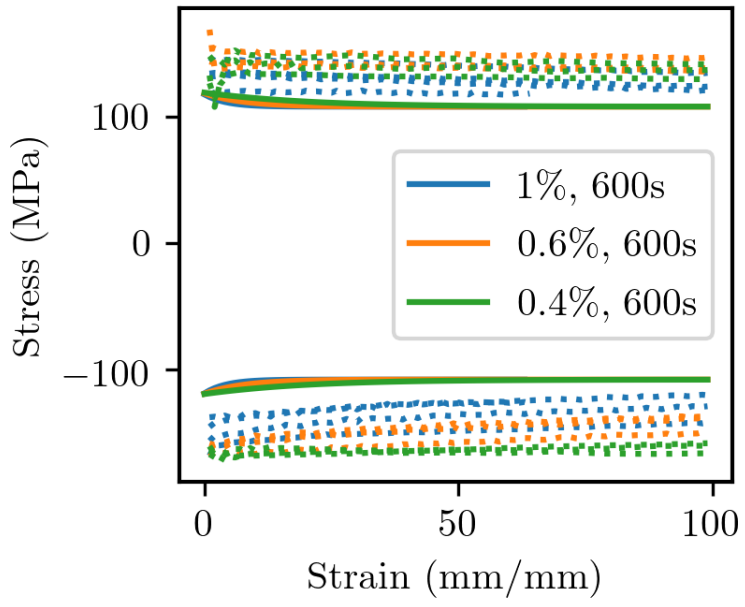

(a) Parameter set "A".

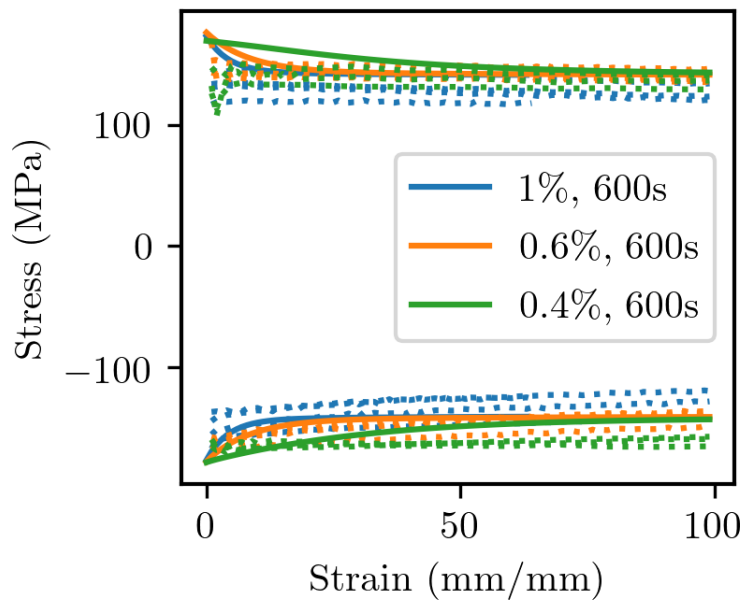

(c) Parameter set "C".

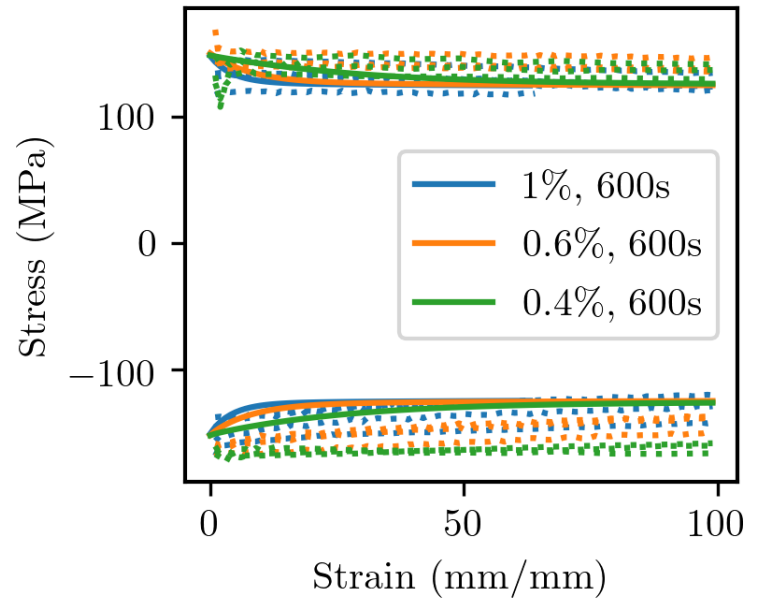

(b) Parameter set "B".

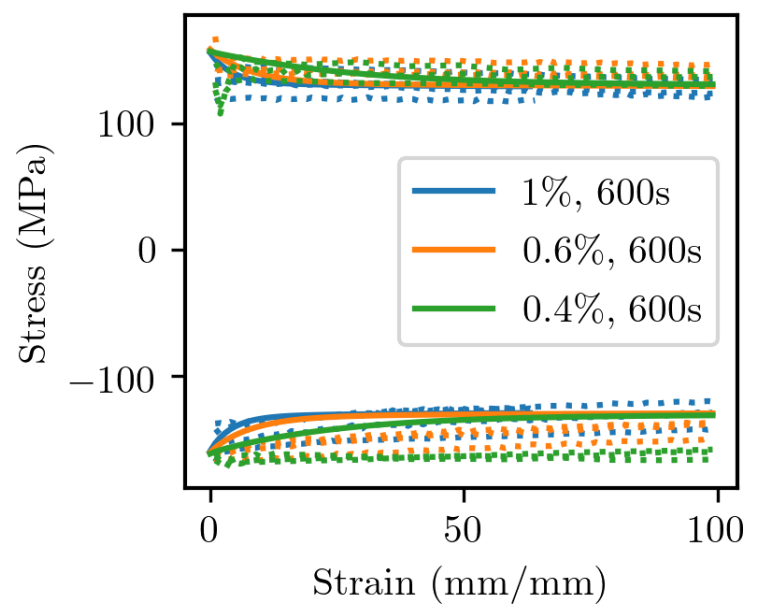

(d) Parameter set "D".

Figure 3.11: Comparison between the model and experimental maximum and minimum stress per cycle for three cyclic tests. The conditions shown in figure legends give the total strain range for fully-reversed, strain-controlled loading and the tensile hold time. All tests were at $950^{\circ} \mathrm{C}$. 


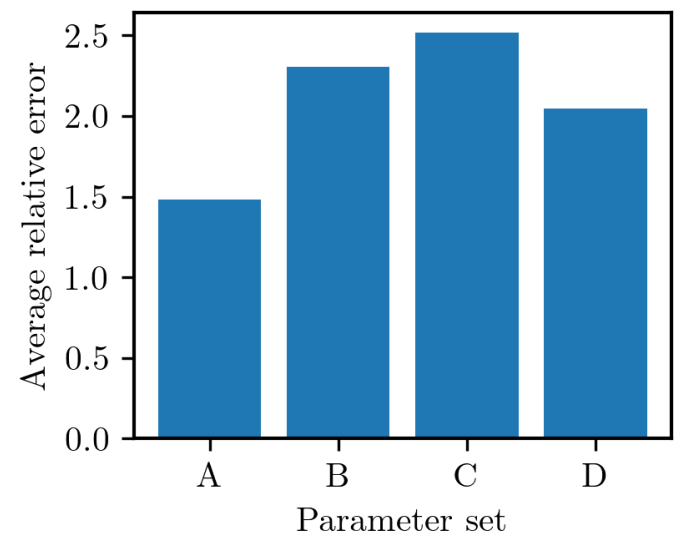

Figure 3.12: Comparison in average mean relative error for the four parameter sets, evaluated over all 32 strain controlled cyclic experiments.

most accurate overall response. Only parameter set "A" reasonably approximates the low temperature uniaxial tension flow curves, though all four models capture the higher temperature tensile data. None of the four parameter sets accurately captures the response of the material under constant stress, creep conditions. All four models underestimate the initial primary creep rate and, for most conditions, the models fail to accurately capture the primary creep rates and the transition to tertiary creep. Based on the past reports, the model was not calibrated against creep data directly. At a minimum then, a new parameter set would need to be developed to better capture this critical aspect of the material response.

The other problem with the Walker model is the model's complexity and the number of (notionally) temperature-dependent parameters. The model has many specialized features beyond a standard Chaboche viscoplastic form aimed at capturing fine details of the material response. Many of these details are not important for the engineering response of a component. A more standard viscoplastic model might capture the Alloy 617 material response with sufficient accuracy with the added benefit of a simpler model form and few configurable parameters. Ease of implementation should be a concern for a model included in the ASME Code, as engineers at a variety of design firms may end up implementing the model.

Overall then, our recommendation is to take the Walker model as a baseline but aim to:

1. Improve the accuracy of the model when compared to the creep rate data;

2. Simplify the model form and reduce the number of configurable parameters;

3. Retain comparable accuracy for the gross features of the cyclic response (ratcheting rates, stress relaxation rates, etc.) while sacrificing some of the fine details of the cyclic stress/strain response.

This model might resemble something more like a standard Chaboche viscoplastic form. However, a few key features of the Walker model, notably the thermal scaling terms and the ability to separately control the hardening/softening of the isotropic hardening and the drag stress, could be retained. 


\section{Conclusions}

This report describes the implementation and evaluation of an existing viscoplastic model for the response of Alloy 617, with the end goal of developing a model to be included in the ASME Boiler \& Pressure Vessel Code. Specifically, the report covers the implementation of the Walker model in the NEML framework and the evaluation of its response against a large database of Alloy 617 test data. While the Walker model has several good features and captures the cyclic stress/strain test data with good accuracy, its treatment of longterm creep deformation requires some improvement. In addition, the model is likely too complicated for inclusion in the ASME Code.

Additional experimental data in the form of lower temperature cyclic tests would be helpful in developing the new, simplified model. One of the disadvantages Walker et al. faced was in the need to develop a model that spanned a wide range of temperatures while only having cyclic test data at a very limited number of temperatures. The broader data set collected as part of the present work does not ameliorate this issue, as the database largely relies on the INL cyclic test data.

Future work will develop a simplified Alloy 617 model and calibrate it against the experimental database described here. This model will retain some of the features of the Walker model, including possibly its treatment of temperature scaling and rate sensitivity. In addition to simplifying the model's mathematical form and reducing the number of temperature-dependent parameters, the new model should focus on better-capturing longterm creep rates, which could be quantified by comparing the time to $1 \%$ accumulated strain predicted by the model to the experimental correlation developed for the Alloy 617 Code Case, and improving the stress relaxation behavior of the model during the hold periods in the creep fatigue data. Ideally, these improvements could be made without compromising the accuracy of the model stress/strain hysteresis loops when simulating the creep-fatigue tests. This new model will then be proposed for incorporation into the ASME Boiler \& Pressure Vessel Code as an amendment to the Alloy 617 Code Case. 



\section{Acknowledgments}

The research was sponsored by the U.S. Department of Energy, under Contract No. DEAC0206CH11357 with Argonne National Laboratory, managed and operated by UChicago Argonne LLC. Programmatic direction was provided by the Office of Nuclear Reactor Deployment of the Office of Nuclear Energy. The authors gratefully acknowledge the support provided by Sue Lesica, Federal Manager, Advanced Materials, Advanced Reactor Technologies (ART) Program, Diana Li, Federal Program Manager, ART Gas-Cooled Reactors (GCR) Campaign, and Gerhard Strydom of Idaho National Laboratory, National Technical Director, ART GCR Campaign. 



\section{Bibliography}

[1] A. K. Dhalla. Recommended practices in elevated temperature design: a compendium of breeder reactor experiences (1970-1987): Volume III - Inelastic analysis. Technical Report 366, 1991.

[2] M. C. Messner, V.-T. Phan, and T.-L. Sham. FY17 Status Report on the Initial Development of a Constitutive Model for Grade 91 Steel. Technical report, Argonne National Laboratory, ANL-ART-93, OSTI 136734, Lemont, IL, 2017.

[3] M. C. Messner, V.-T. Phan, and T.-L. Sham. A Unified Inelastic Constitutive Model for the Average Engineering Response of Grade 91 Steel. In Proceedings of the 2018 ASME Pressure Vessels and Piping Conference. PVP2018-84104, 2018.

[4] M. C. Messner, V.-T. Phan, and T.-L. Sham. Development of Grade 91 inelastic model for incorporation in ASME Division 5. Technical report, Argonne National Laboratory, ANL-ART-137, OSTI 146678, Lemont, IL, 2018.

[5] M. C. Messner, V.-T. Phan, and T.-L. Sham. Development of the Technical Basis of a Unified Viscoplastic Model of 316H Stainless Steel for Incorporation into ASME Division 5. Technical report, Argonne National Laboratory, ANL-ART-166, Lemont, IL, 2019.

[6] M. C. Messner and T.-L. Sham. Inelastic analysis procedure based on the Grade 91 unified viscoplastic constitutive model for ASME implementation. Technical report, Argonne National Laboratory, ANL-ART-167, Lemont, IL, 2019.

[7] T.-L. Sham and Kevin P. Walker. Preliminary Development of a Unified Viscoplastic Constitutive Model for Alloy 617 With Special Reference to Long Term Creep Behavior. In Fourth International Topical Meeting on High Temperature Reactor Technology, pages 81-89, 2008.

[8] Personal communication with T.-L. Sham, 2020.

[9] K. P. Walker and T. L. Sham. A fixed-point iteration method with quadratic convergence. Journal of Applied Mechanics, Transactions ASME, 79(3):1-10, 2012. ISSN 00218936. doi: 10.1115/1.4005878.

[10] L. C. Carroll, C. Cabet, and R. Wright. The Role of Environment on High Temperature Creep-Fatigue of Alloy 617. In ASME 2010 Pressure Vessel and Piping Conference, pages PVP2010-26126, 2010.

[11] M C Messner, V Phan, and T Sham. International Journal of Pressure Vessels and Piping Evaluating and modeling rate sensitivity in advanced reactor structural materials : 316H , Gr . 91, and A617. International Journal of Pressure Vessels and Piping, 178:103997, 2019. ISSN 0308-0161. doi: 10.1016/j.ijpvp.2019.103997. URL https://doi.org/10.1016/j.ijpvp.2019.103997.

[12] K. P. Walker and E. Krempl. An implicit functional representation of stress-strain behavior. Mechanics Research Communications, 5(4):185-190, 1978. 
[13] Yanli Wang, Robert I. Jetter, and T.-L. Sham. Application of Simplified Model Tests to Alloy 617 Creep-Fatigue Damage Evaluation. Technical report, Oak Ridge National Laboratory, ORNL/TM-2014/360, Oak Ridge, TN, 2014.

[14] Vivek Agarwal, Richard N Wright, and Timothy Roney. Prediction of the Creep-Fatigue Lifetime of Alloy 617 : an Application of Non-Destructive Evaluation and Information Integration. In Proceedings of the 22nd Conference on Structural Mechanics in Reactor Technology, 2013.

[15] Walter Osthoff, Hans Schuster, Philip J. Ennis, and Hubertus Nickel. Creep and relaxation behavior of Inconel-617. Nuclear Technology, 66(2), 1984. ISSN 00295450. URL http://www.osti.gov/energycitations/product.biblio.jsp?osti_id=6469226.

[16] U F Kocks. Realistic constitutive relations for metal plasticity. Materials Science and Engineering A, 317:181-187, 2001.

[17] H Mecking, B Nicklas, N. Zarubova, and U F Kocks. A "universal" temperature scale for plastic flow. Acta Metallurgica, 34(3):527-535, 1986. 



\section{Argonne}

\section{Applied Materials Division}

Argonne National Laboratory

9700 South Cass Avenue, Bldg. 212

Argonne, IL 60439

www.anl.gov 